\title{
Review \\ The Effects of Physical Activity on the Aging of Circulating Immune Cells in Humans: A Systematic Review
}

\author{
Lara Brauer, Karsten Krüger*, Christopher Weyh (D) and Katharina Alack \\ Department of Exercise Physiology and Sports Therapy, Institute of Sports Sciences, \\ Justus-Liebig-University Giessen, 35394 Giessen, Germany; lara.brauer@sport.uni-giessen.de (L.B.); \\ christopher.weyh@sport.uni-giessen.de (C.W.); katharina.alack@sport.uni-giessen.de (K.A.) \\ * Correspondence: karsten.krueger@sport.uni-giessen.de
}

\begin{abstract}
Age-induced cellular senescence leads to a decline in efficacy of immune response and an increase in morbidity and mortality. Physical activity may be an intervention to slow down or reverse this process for elderly individuals or even delay it via enhanced activity over their lifespan. The aim of this systematic review was to analyze and discuss the current evidence of the effects of physical activity on senescence in leukocyte subpopulations. Two electronic databases (PubMed, Web of Science) were scanned in July 2020. Studies performing endurance or resistance exercise programs and investigating leukocytes of healthy, particularly elderly subjects were included. Nine human studies were identified, including a total of 440 participants, of which two studies examined different types of exercise training retrospectively, three conducted resistance exercise, three endurance exercise, and one endurance vs. resistance training. Results revealed that exercise training increased the naïve subsets of peripheral T-helper cells and cytotoxic T-cells, whereas the senescent and effector memory T-cells re-expresses CD45RA (TEMRA) subsets decreased. Moreover, the percentage of T-helper- compared to cytotoxic T-cells increased. The results suggest that physical activity reduces or slows down cellular immunosenescence. Endurance exercise seems to affect cellular senescence in a more positive way than resistance training. However, training contents and sex also influence senescent cells. Explicit mechanisms need to be clarified.
\end{abstract}

Weyh, C.; Alack, K. The Effects of Physical Activity on the Aging of Circulating Immune Cells in Humans: A Systematic Review. Immuno 2021, 1 , 132-159. https://doi.org/10.3390/ immuno1030009

Academic Editor: Jun Sik Lee

Received: 3 May 2021

Accepted: 21 June 2021

Published: 24 June 2021

Publisher's Note: MDPI stays neutral with regard to jurisdictional claims in published maps and institutional affiliations.

Copyright: (c) 2021 by the authors. Licensee MDPI, Basel, Switzerland. This article is an open access article distributed under the terms and conditions of the Creative Commons Attribution (CC BY) license (https:// creativecommons.org/licenses/by/ $4.0 /)$.

\section{Introduction}

Like all other organs and tissues, the immune system is also subject to an aging process. Besides an adaptive remodeling process, the ability to react adequately against pathogens decreases. [1]. This dysfunction is reflected by a permanent state of mild activation and a decreased amplitude of the dynamic response when stimulated [1]. A key feature of this immunological remodeling process is a change in the composition of leukocyte subpopulations in the blood. [2]. The investigated severe changes of the immune response during the aging process are termed immunosenescence [3]. The stable cell cycle arrest in immune cells is defined as cellular senescence and stands in connection with phenotypic changes [4]. These phenotypic changes in the sense of differentiation and often associated functional impairment are the focus of this article. Among these changes are shifts in T-cell subsets, cytokine secretion pattern, and cell replication capacity, as well as reductions in antibody production. Altogether, this results in a pro-inflammatory state called 'inflamm-aging', which is characterized by a reduced capacity to respond to pathogens or antigens [3]. These changes induced by immunosenescence are associated with an increase in the incidence and severity of infectious diseases and thereby higher morbidity and mortality rates and poorer vaccine response in elderly humans $[3,5,6]$.

Physical activity (PA) may be an intervention to slow down or reverse cellular senescence. Regular exercise affects the function of different leukocyte subpopulations, specifically the composition of T-cell subsets [7]. Additionally, PA has an effect on our genome 
and its proportion, which has evolved to optimize aerobic metabolism, especially in an environment of food scarcity [4]. In particular, exercise affected the proportion of T-cell subpopulations and T-cell proliferative capacity [5]. Lower levels of circulating inflammatory cytokines, and therefore a lower inflamm-aging state, increased neutrophil phagocytic activity, greater natural killer cell (NK cell) cytotoxic activity, and longer telomeres in leukocytes of aged humans indicate that regular PA seems capable to improve the immune response and delays cellular senescence. However, data collected from the literature regarding the effects of PA on age-related changes in the immune system are conflicting and limited, and existing research shows either a benefit or declares a lack of evidence [3]. The reported positive effects of PA on cellular senescence in cross-sectional studies are mostly not reported in longitudinal exercise training studies [5]. Consequently, the intensity of exercise-induced immune changes in elderly individuals is diverse, possibly due to differences in methodology [8]. A positive influence of PA on cellular senescence could potentially be a cost-effective therapy to improve human quality of life [3]. Therefore, exercise immunology is a current topic with much developmental potential for research.

The present systematic review addresses the effects of different types of PA on cellular senescence in immune cell subpopulations. The aim was to gather the current evidence for cellular senescence in relation to endurance and resistance exercise training. The comparison of active with sedentary subjects, baseline data, or another exercise intervention group at a different intensity, as well as the comparison of old and young subjects, is accepted as appropriate for inclusion. All subpopulations of leukocytes, despite the rare studied eosinophilic and basophilic granulocyte populations, are considered for a global view on immune system alterations induced by PA. The relationship of PA and the cellular senescence of leukocytes were systematically analyzed to develop a deeper understanding of immunosenescence and to resolve whether PA can improve immunological space in elderly individuals by inhibiting the reduction in the immune system defense.

\section{Cellular Senescence}

\subsection{Classification and Mechanisms of Cellular Senescence}

As humans age, their capability to respond to and eliminate pathogens decreases, leading to a greater incidence and severity of diseases. In addition, the response and efficacy of vaccines are frequently decreased in elderly individuals. Increased sensitivity to infections as well as reduced protection after vaccination mirror the impact of age-related changes to the immune system [9].

A decline in immune function is one of the most acknowledged the consequence of aging. Elderly individuals do not respond as efficiently as younger individuals to novel or previously exposed antigens. Aging affects the immune system globally, for example, the rate of naïve $\mathrm{B}$ - and $\mathrm{T}$-cell production as well as the constitution and quality of the mature lymphocyte pool [2]. Senescence (Latin: senescere, to grow old) is a process leading to functional declines and simultaneously an increase in vulnerability to a wide range of diseases that can lead as far as the death of an organism [10]. Cellular senescence is a term involving irreversible replicative arrest, apoptosis resistance, frequently increased protein synthesis, metabolic shifts, and a greater reactive oxygen species (ROS) generation. It is a mechanism underlying aging and age-related conditions, where cells lose the ability to divide and damage close-by cells due to the factors they secrete, labeled as the senescenceassociated secretory phenotype (SASP) [11,12]. In cell cultures, senescent cells can be recognized by phenotypic characteristics such as increased cell size, flattened and irregular shape, multi-nucleation, and cytoplasmic vacuolation [10]. This irreversible arrest of cell growth and affected function of mitochondria, endoplasmic reticulum, and lysosomes are underlying molecular mechanisms involving, among other things, tumor suppressor genes p16 and p53. Replicative senescence is associated with telomere dysfunction and cell arrest at the G1/S or G2/M boundary, hence permanent exit from the cell cycle $[13,14]$. 


\subsection{The Effects Of aging on Leukocyte Subsets}

The totality of changes in immune parameters with aging is termed immunosenescence [15]. Immunosenescence can be described as a remodeling of immune function during the aging process. It affects all components of the immune system; however, the decline in adaptive immunity ( $\mathrm{T}$ - and B-lymphocytes and their subsets) is more clearly defined, unlike the effects of aging on innate immunity. Adaptive immunity seems to have the greatest modification and contribution in degrading the immune system in the elderly. The innate immune system, mainly represented by monocytes, NK cells, and dendritic cells (DCs), is partially preserved during aging [16,17]. An overview of the most prominent changes regarding innate and adaptive immunity is presented in Table 1.

Table 1. Summary of selected age-related immunological changes in innate and adaptive immunity.

\begin{tabular}{cccc}
\hline Innate Immunity & Changes & Adaptive Immunity & Changes \\
\hline Phagocytosis & $\downarrow \leftrightarrow$ & Nä̈e cell number & $\downarrow$ \\
Free radical production & $\uparrow \downarrow$ & Memory cell number & $\uparrow$ \\
Chemotaxis & $\downarrow$ & T-regulatory cell number & $\uparrow$ \\
Cytokine production & $\uparrow$ & T-regulatory cell function & $\downarrow$ \\
Myeloid cell number & $\uparrow$ & Proliferation & $\downarrow$ \\
& & IL-2 & $\downarrow$ \\
& & B-regulatory cell number/function & $\downarrow$ \\
& B-cell immunoglobulin production & $\downarrow$ \\
& B-cell autoantibody production & $\uparrow$ \\
\hline
\end{tabular}

$\downarrow$ : decreased; $\uparrow:$ increased; $\leftrightarrow$ : no change; IL-2: Interleukin 2. Modified according to Fulop et al. [15].

Age-related cellular changes are additionally accompanied by modifications of the cytokine network. IL-2 shows a modest decrease, whereas there is a two- to four-fold increase in circulating cytokines, proteases, and chemokines, such as TNF $\alpha, \mathrm{IL}-6$, and Creactive protein (CRP). This general activity of the inflammatory network associated with age is termed 'inflamm-aging' [18]. Inflamm-aging is characterized by pro-inflammatory biomarkers and associated with other immunological risks, like smoking, obesity, and multiple chronic diseases $[18,19]$. Stressor pathways in chronic inflammation such as ROS production and SASP are mostly mediated via the transcript factor NF- $\mathrm{KB}$ (nuclear factor kappa-light-chain-enhancer of activated B-cells). These stressors, like obesity, mitochondrial dysfunction, and genomic instability, cause a pro-inflammatory environment and therefore trigger cellular senescence and immune cell dysfunction. The mechanisms of aging and other stressors leading to a pro-inflammatory environment and, therefore, to inflamm-aging, are illustrated in Figure 1. 


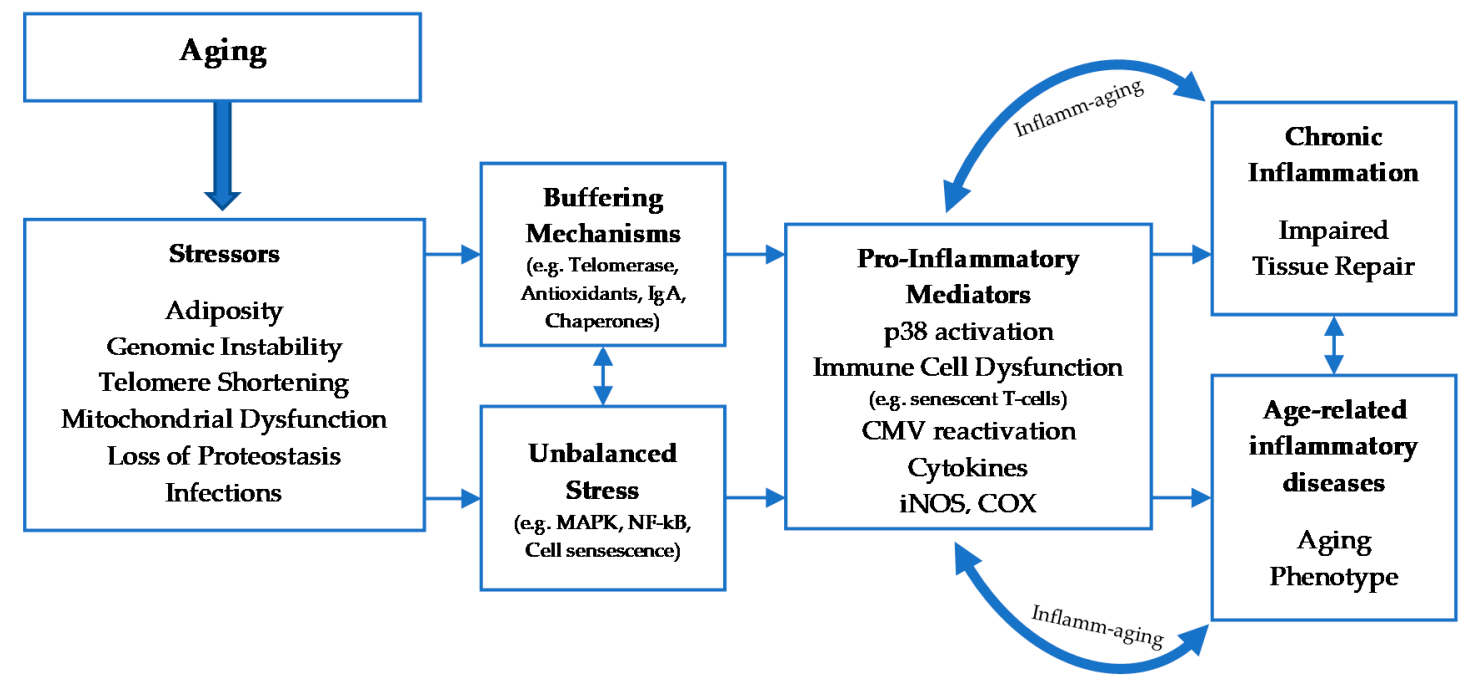

Figure 1. Mechanisms leading to a pro-inflammatory state with aging and a vicious cycle of inflamm-aging. Ageinduced stressors that can potentially trigger inflammation, as well as unbalanced stress like cell senescence, are normally downregulated by buffering mechanisms. If these mechanisms fail, the existing stress leads to the production of proinflammatory mediators and disruption of immune cells, which is conducive to a chronic inflammation status and age-related inflammatory diseases. This then again deteriorates the inflamm-aging process in a positive feedback loop through an increase in circulating inflammatory mediators. CMV: Cytomegalovirus; COX: Cyclooxygenase; IgA: Immunoglobulin A; iNOS: inducible nitric oxide synthase; MAPK: Mitogen-activated protein kinase; NF- $\mathrm{B}$ : nuclear factor kappa-light-chainenhancer of activated B-cells. Modified according to Bektas et al. [1] and Macaulay et al. [20].

\subsection{Physical Activity and Immune System}

In the present systematic review, PA refers to all activities associated with movement, sports, or fitness, which includes a level of activity to remain healthy as recommended and published by the World Health Organization [21]. Aerobic, anaerobic, or resistance exercise training is included in this review.

There are several accepted findings regarding the immune system and exercise. The main finding of those studies is the increased infection risk after acute high-intensity exercise, the temporary reduction in salivary Immunoglobulin A (IgA) levels which leads to a higher risk of infection and a decrease in the numbers of immune cells in the peripheral blood resulting in a period of immune suppression [22]. These observations resulted in the 'open-window theory' and the associated connection of the incidence of infections of the upper respiratory tract (URTI) and PA in a 'J'-shaped model. Moderate levels of PA are associated with a low risk of URTI, whereas highly active individuals such as elite endurance athletes have an increased frequency of URTI after single bouts of ultraendurance exercise or during intensive training periods. Inactive individuals are at high risk of URTI as well [16,23-25].

It is well-established that acute and chronic (persistent) exercise changes the innate and the adaptive immune response by immune cell count and function $[16,22,26]$. In this connection, the mode and intensity of exercise or PA play an important role in the effect of the immune system [26]. The benefits of regular exercise are dose-dependent. Higher levels of moderate-to-vigorous exercise $(\geq 450 \mathrm{~min} /$ week) are associated with longevity. However, elite athletes, who sustain the highest possible exercise levels, also usually live considerably longer than the general population [4]. In contrast, a sedentary, inactive lifestyle is associated with immune depression and can result in chronic inflammation [27]. Chronic inflammation is linked with insulin resistance, atherosclerosis, neurodegeneration, and tumor growth [16].

Regular PA has an impact on neutrophil function, NK cell cytotoxicity, naïve T-cells, regulatory T-cells (Treg cells), and TEMRA cells $[7,16]$. In this connection, it is important to differentiate between moderate training intensities and intense training intensities 
in regular exercise for athletes. During a moderate training cycle, there is apparently an increase in neutrophil function, naïve T-cells, and Treg cells, whereas TEMRA cells decrease [7]. Regarding NK cell cytotoxicity, studies show controversial results (increase or no change). Intense training cycles seem to lead to an increase in Treg and TEMRA cells and a decrease in naïve T-cells. The neutrophil function is also reduced. These results reveal that athletes seem to be at a greater risk of infection in response to strenuous training loads compared to moderate training loads (e.g., in pre-season), which appears to promote immune functionality [7].

\section{Methods}

\subsection{Search Strategy}

A search strategy was generated to undertake systematic literature research. The electronic databases PubMed, providing research from the Medline database, and Web of Science were considered as suitable regarding the topic of this review. In preparation, the Preferred Reporting Items for Systematic Reviews and Meta-Analysis (PRISMA) [28] were screened and used as guidance. The systematic search took place between 26-30 July 2020. An overview of the used headings and search terms is shown in Table 2. Search terms were identified by using synonyms and umbrella terms for each heading. The headings were combined by the Boolean search operator 'OR', search terms were combined by the Boolean search operator 'AND'. Additionally, diseases that have extensive effects on the immune system (HIV, cancer, and diabetes mellitus) and all topics related to vaccination were excluded in advance through the Boolean search operator ' $N O T^{\prime}$. The time span was set for articles published between 2000-2020, and article type was filtered for clinical trials and randomized controlled trials (RCTs) in PubMed. Reviews and Meeting Abstracts were excluded beforehand in Web of Science. Limitations were made on English and German language. After the first perusal of identified studies and, in addition, reviews and articles found during incorporating into the topic, a deeper understanding of the subject was gained. Thus, suitable eligibility criteria could be defined. Due to a lack of appropriate exercise-related studies examining eosinophils and basophils, these cell populations were excluded in advance.

Table 2. Overview of the search terms used for this systematic review.

\begin{tabular}{cc}
\hline Headings & Search Terms \\
\hline Physical activity & $\begin{array}{c}\text { exercise, physical exercise, physical activity, physical fitness, } \\
\text { training, exercise training, activity, aerobic training, aerobic } \\
\text { fitness, strength training, resistance training, physical } \\
\text { endurance, and strength endurance training }\end{array}$ \\
\hline Cellular senescence & $\begin{array}{c}\text { senescence, cellular senescence, cell aging, senescent cells, } \\
\text { immunosenescence, and aging }\end{array}$ \\
\hline Leukocyte subpopulations & $\begin{array}{c}\text { leukocyte, leukocytes, leukocyte subpopulations, leukocyte } \\
\text { populations, lymphocytes, T-cells, B-cells, monocytes, } \\
\text { macrophages, dendritic cells, natural killer cells, } \\
\text { neutrophils, and immune system }\end{array}$ \\
\hline
\end{tabular}

Headings were combined by the Boolean search operator 'AND'. Search terms were combined by the Boolean search operator 'OR'.

\subsection{Eligibilty Criteria}

First, duplicates of the eligible studies were excluded. Articles had titles screened and were excluded if there was no exercise program included and focus set on ill subjects or nutritional strategies. Then, remaining articles were screened for title/abstract and excluded if there was no open access, other cell types than leukocytes analyzed, no acute or chronic physical activity performed in comparison to sedentary subjects, or focus set on the functional response of skeletal muscle on exercise. Only RCTs, non-RCTs (non-randomized or non-controlled trials), and observational studies were included. Meeting abstracts, case 
reports, conference papers, and study protocols were excluded. Full texts of the remaining articles were scanned for final inclusion or exclusion. Studies not matching all of the inclusion criteria were found unsuitable and were excluded, as well as by the exclusion criteria. Inclusion and exclusion criteria are shown in Table 3.

Table 3. Eligibility criteria for inclusion and exclusion of studies.

\begin{tabular}{|c|c|}
\hline Inclusion Criteria & Exclusion Criteria \\
\hline $\begin{array}{l}\text { healthy subjects (no manifested chronic illness, } \\
\text { immune disease or no acute infection) }\end{array}$ & $\begin{array}{l}\text { ill subjects(especially } \mathrm{HIV}+\text {, cancer, or } \\
\text { diagnosed DM) }\end{array}$ \\
\hline human study & animal study \\
\hline $\begin{array}{c}\text { elderly subjects }(\geq 50) \text {; elderly compared to } \\
\text { young subjects ( } 18-85 \text { years) }\end{array}$ & $\begin{array}{l}\text { younger subjects }(<50 \text { years); except elderly } \\
\text { ( }>85 \text { years) compared to young subjects }\end{array}$ \\
\hline $\begin{array}{l}\text { acute exercise, if not at least lifelong training } \\
\text { and/or } 2 \text { cohorts (trained/sedentary or } \\
\text { aerobic/resistance exercise or } \\
\text { pre-/post-intervention) }\end{array}$ & $\begin{array}{c}\text { no exercise, no comparison of sedentary and } \\
\text { active subjects, or no sufficient survey of } \\
\text { physical activity }\end{array}$ \\
\hline $\begin{array}{l}\text { some kind of control group or } 2 \text { intervention } \\
\text { groups or longitudinal analysis }\end{array}$ & $\begin{array}{l}\text { no control group, only one intervention group, } \\
\text { or cross-sectional analysis without controls }\end{array}$ \\
\hline aerobic, anaerobic, or resistance exercise & $\begin{array}{l}\text { exercise with a focus on coordination or } \\
\text { flexibility (as intervention group) }\end{array}$ \\
\hline $\begin{array}{c}\text { investigation of any kind of leukocyte } \\
\text { subpopulations (except eosinophilic and } \\
\text { basophilic granulocytes) or telomere length } \\
\text { through blood samples }\end{array}$ & $\begin{array}{l}\text { eosinophilic and basophilic granulocytes, } \\
\text { change in insulin sensitivity, ROS production, } \\
\text { DNA damage, tissue biopsy, macrophages in } \\
\text { skeletal muscle as an indicator for } \\
\text { rehabilitation after exercise }\end{array}$ \\
\hline $\begin{array}{l}\text { effect of acute or chronic physical activity on } \\
\text { leukocyte subpopulations }\end{array}$ & $\begin{array}{l}\text { effects of vaccination or nutritional strategies } \\
\text { (like CR or consumption of } \\
\text { supplements/vitamins) on immune response }\end{array}$ \\
\hline
\end{tabular}

CR: calorie restriction; DM: diabetes mellitus; HIV+: human immunodeficiency virus positive; ROS: reactive oxygen species.

In general, the aim of the present review was to identify comparable high-quality studies to gain evidence-based results. For this reason, only human studies were included.

Furthermore, only older or elderly subjects ( $\geq 50-85$ years) should be investigated because, at the beginning of the sixth decade of life, the human immune system starts to undergo crucial aging-related changes. These changes continue to the state of immunosenescence [29]. It needs to be clarified that the terms 'older', 'aged', and 'elderly' used in this review refer to named age range of $\geq 50-85$ years and concern to the immunological age and not the general biological age of humans.

Studies investigating trained versus untrained subjects or elderly against young test persons were considered. PA was understood as either aerobic or anaerobic endurance, as well as resistance exercise or strength training. No mixed interventions analyzing endurance and resistance exercise simultaneously in the same group were investigated. The purpose was to compare the different types of exercise intervention and to identify a superior exercise mode to prevent or slow down immunosenescence. Thus, studies involving lifelong training, sex differences, and high-intensity exercise were accepted as eligible [5].

\subsubsection{Inclusion of Studies}

After defining the search strategy, including databases, headings, keywords selection, and inclusion plus exclusion criteria, systematic research was conducted. The identification and screening process was conducted as shown in the flow chart in Figure 2. Though, the research process showed that there is a lack of adequate and properly executed interventions regarding PA and senescence of leukocyte subpopulations, especially in RCTs. 
Identified articles were thoroughly screened, and in the end, a total of six $(n=6)$ articles were accepted as eligible to be assessed in this review. Following, the reference list from these six included articles was screened manually to identify additional records. Using this approach, three $(n=3)$ articles were determined as eligible to be included and assessed. A detailed summary of considered articles, including database and title/year can be seen in the Appendix A (Table A1).

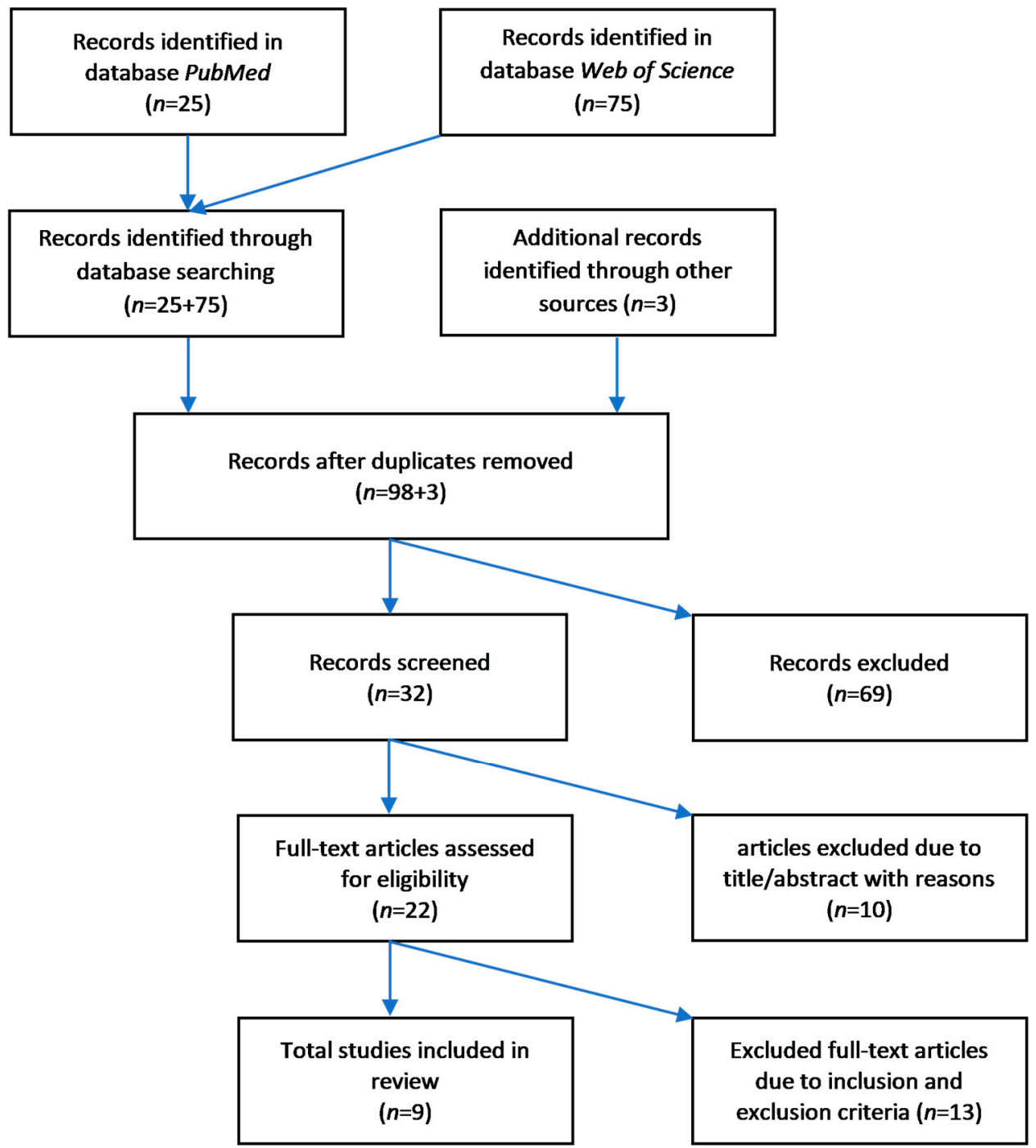

Figure 2. Flow chart of the inclusion process of studies used in this review. A total of $n=9$ studies were finally included and considered for this review. Modified according to Moher et al. [28].

\subsubsection{Exclusion of Studies}

The exclusion of full-text articles was performed after perusal and applying exclusion criteria to test the eligibility of found articles. In total, $n=13$ studies were excluded in the last screening step due to the reasons named in Table 4. These studies were excluded due to methodological issues and not meeting the inclusion criteria. Furthermore, an adequate methodological description and a reproducible approach had to exist; otherwise, the study was excluded as well. 
Table 4. Excluded studies with named reasons $(n=13)$.

\begin{tabular}{cc}
\hline Author (Year) & Reason for Exclusion \\
\hline [30] Chilton et al. (2014) & outcome measures $\rightarrow$ focus on miRNA, SIRT, TERT, and TL \\
\hline [31] Cosgrove et al. (2012) & cohort $\rightarrow$ age of subjects does not meet inclusion criteria \\
$(<50$ years)
\end{tabular}

The study of Dinh et al. [32] had to be excluded because the same cohort was already analyzed in the included study of Dinh et al. [33] and published by the same author. Besides, the focus was set mainly on CMV infection and, thus, did not meet the inclusion criteria.

\subsection{Study Quality Assessment}

The study quality for each of the nine extracted articles was assessed depending on the design. For RCTs, the RoB2 Tool for randomized controlled trials, developed by Higgins et al. [44] for the Cochrane Collaboration, was used to analyze the risk of bias. For nonrandomized clinical trials and observational studies, the risk of bias was assessed using the ROBINS-I, a tool developed by Sterne et al. [45]. Those risk of bias assessment tools are well established and have already been used in previous systematic reviews about physical activity and its effect on different kinds of leukocyte functions, for example, in Palmowski et al. [46]. The ROBINS-I tool offers a systematic way to organize, analyze and present potential bias domains and content for non-RCTs, non-controlled trials, or observational studies [45]. The tool comprises different categories of risk of bias. Content questions with potential biases and an overview of the different categories used for the ROBINS-I tool are shown in Table 5. The RoB2 Tool was developed for randomized parallel-group trials. It contains 5 different domains which have potential for biases: bias arising from the randomization process, bias due to deviations from intended interventions, bias due to missing outcome data, bias in the measurement of the outcome, and bias in the selection of the reported result [44]. Both assessments categorize the studies in different kinds of risk states. The RoB2 Tool subclassifies into the labels 'high risk', 'moderate risk', 'low risk', 
or 'unclear risk'. The ROBINS-I Tool subclassifies into 'low risk', 'moderate risk', 'serious risk', 'critical risk', and 'unclear risk'. The label 'unclear risk' is used in both tools if there is unclear or insufficient information to allow a judgment.

Table 5. Domains and content questions used to analyze the quality of non-RCT studies with the ROBINS-I.

\section{Results}

The specified research process, perusal, as well as inclusion or exclusion of studies, resulted in a total of nine studies included in this review. Three of those studies were found in PubMed [33,47,48], three in Web of Science [49-51], and three of them were additionally found after perusal of those six studies and scanning the reference lists [52-54]. Three studies were RCTs, four studies were non-RCTs (not randomized or not controlled), and two were cohort studies. All of them investigated humans. All the discovered studies were written in English. They were published between 2007 and 2019. In two studies, there were partly the same authors involved (A.B. Bigley and R.J. Simpson) [49,52]. Moreover, in one study, two of the authors of this review were incorporated [50]. The characteristics of included studies are summarized in Table 6.

Adding the sample size for each study up, altogether, a total of 440 participants (male and female) were investigated. Eight studies comprise elderly participants, two of them compare those elderly subjects with young ones. The remaining one study investigates young adults [52]. Nearly all studies provided information about the sex of participants; only Abd El-Kader et al. [48] and Silva et al. [51] did not consider sex in their investigations. Three studies only incorporated female participants, two just included male subjects, and the remaining two investigated both sexes-one study investigated in particular sex influences on senescent T-cells [52]. 
Table 6. Characteristics of included exercise training studies $(n=9)$.

\begin{tabular}{|c|c|c|c|c|}
\hline Author (Year) & Design & Cohort & Duration & Exercise \\
\hline $\begin{array}{l}\text { [48] Abd El-Kader et al. } \\
\text { (2018) }\end{array}$ & $\begin{array}{c}\text { Non-RCT } \\
\text { (no CG, } 2 \text { IG) }\end{array}$ & $\begin{array}{l}60 \text { sedentary elderly subjects } \\
61-67 \text { yrs } \\
\text { aerobic group }(n=30) \\
\text { resistance group }(n=30)\end{array}$ & $\begin{array}{c}6 \text { months } \\
3 \text { days/week }\end{array}$ & $\begin{array}{c}\text { aerobic exercise } \\
40 \text { min treadmill } \\
\text { resistance exercise } \\
40 \text { min, } 9 \text { resistance machines } \\
\text { supervised, monitored }\end{array}$ \\
\hline [52] Brown et al. (2014) & Non-RCT & $\begin{array}{c}32 \text { adults } \\
\text { trained soccer players }(n=16) \\
8 \text { males, } 8 \text { females, } 18.3 \pm 1.7 \text { yrs } \\
\text { untrained controls }(n=16) \\
8 \text { males, } 8 \text { females, } 19.3 \pm 2.0 \text { yrs }\end{array}$ & acute exercise & $\begin{array}{c}\text { treadmill running test } \\
\text { (to volitional exhaustion) } \\
\text { starting at } 10 \mathrm{~km} / \mathrm{h} \text { (males) / } \\
8 \mathrm{~km} / \mathrm{h} \text { (females) } \\
1 \mathrm{~km} / \mathrm{h} \text { every } 3 \mathrm{~min}\end{array}$ \\
\hline [33] Dinh et al. (2019) & $\begin{array}{c}\mathrm{RCT} \\
(2 \mathrm{IG}, 1 \mathrm{CG})\end{array}$ & $\begin{array}{c}100 \text { women }(\geq 65 \text { yrs }) \\
\text { not performing for past } \\
6 \text { months } \\
\text { IST }(n=31,69.18 \pm 5.12 \text { yrs }) \\
\text { SET }(n=33,69.02 \pm 6.05 \text { yrs }) \\
\text { CON }(n=36,70.31 \pm 5.15 \text { yrs })\end{array}$ & $\begin{array}{c}6 \text { weeks } \\
\text { 2-3 times/week }\end{array}$ & $\begin{array}{c}\text { IST } \\
3 \times 10 \text { reps at } 80 \% 1 \mathrm{RM}, 1 \mathrm{~min} \\
\text { rest } \\
\text { SET } \\
2 \times 30 \text { reps at } 40 \% 1 \mathrm{RM}, 1 \mathrm{~min} \\
\text { rest } \\
\text { CON (stretching) } \\
3 \times 10-12 \text { stretching exercises, } \\
\text { passive-static }(30 \mathrm{~s})\end{array}$ \\
\hline $\begin{array}{l}\text { [54] Moro-García et al. } \\
\text { (2014) }\end{array}$ & $\begin{array}{l}\text { retrospective } \\
\text { cohort study }\end{array}$ & $\begin{array}{l}95 \text { adults } \\
32.6 \pm 9.9-75.5 \pm 4.2 \text { yrs } \\
\text { young non-athletes }(n=30) \\
\text { young athletes }(n=27) \\
\text { elderly non-athletes }(n=26) \\
\text { elderly athletes }(n=12)\end{array}$ & $\begin{array}{c}\text { no acute exercise } \\
\text { subjects not performing } 18 \mathrm{~h} \\
\text { before blood samples were } \\
\text { taken }\end{array}$ & $\begin{array}{l}\text { young athletes } \\
\text { water rowing, running, } \\
\text { resistance training } \\
6.2 \pm 1 \text { days a week } \\
125.3 \pm 41.5 \text { min a day } \\
\text { for } 13 \pm 4.2 \text { yrs } \\
\text { elderly athletes } \\
\text { endurance, stretching, body } \\
\text { core, resistance training }\end{array}$ \\
\hline [47] Neves et al. (2009) & $\begin{array}{c}\mathrm{RCT} \\
(2 \mathrm{IG}, 1 \mathrm{CG})\end{array}$ & $\begin{array}{c}15 \text { elderly women } \\
67.5 \pm 3.9 \text { yrs } \\
2 \text { resistance exercise sessions } \\
1 \text { control session }\end{array}$ & $\begin{array}{c}\text { acute exercise } \\
4 \text { measurement moments } \\
\mathrm{T} 1, \mathrm{~T} 2, \mathrm{~T} 3 \text {, and T4 }\end{array}$ & $\begin{array}{c}\text { resistance exercise session } \\
50 \% 1 \mathrm{RM} \\
80 \% 1 \mathrm{RM} \\
\text { control session }\end{array}$ \\
\hline [50] Philippe et al. (2019) & $\begin{array}{c}\text { Non-RCT } \\
\text { (no CG, } 2 \text { IG) }\end{array}$ & $\begin{array}{c}16 \text { male older adults } \\
56.9 \pm 5.1 \text { yrs } \\
\text { CE }(n=8) ; \text { EE: }(n=8) \\
\text { Pre-diabetic subjects (IGT) }\end{array}$ & $\begin{array}{c}3 \text { weeks } \\
3 \text { days / week }(M, W \text {, and } F)\end{array}$ & $\begin{array}{l}\text { walking exercise } \\
\text { CE (uphill) } \\
\text { EE (downhill) }\end{array}$ \\
\hline [49] Pistillo et al. (2013) & Non-RCT & $\begin{array}{l}34 \text { physicaly active males } \\
\text { young males ( } n=17,23-35 \text { yrs) } \\
\text { older males ( } n=17,50-64 \text { yrs) }\end{array}$ & $\begin{array}{c}\text { acute exercise } \\
\text { pre and post blood samples }\end{array}$ & $\begin{array}{c}\text { cycling exercise } \\
30 \text { min cycle ergometer at } \\
80-85 \% \text { PP }\end{array}$ \\
\hline [53] Raso et al. (2007) & $\begin{array}{c}\mathrm{RCT} \\
(\mathrm{IG}, \mathrm{CG})\end{array}$ & $\begin{array}{l}42 \text { healthy sedentary females } \\
60-77 \text { yrs } \\
\text { IG, CG }\end{array}$ & $\begin{array}{l}12 \text { months } \\
3 \times / \text { week }\end{array}$ & $\begin{array}{l}\text { resistance training program } \\
\text { light to moderate intensity, } \\
\text { supervised } \\
3 \times 12 \text { reps at } 54.9 \% \pm 2.4 \% \\
1 \mathrm{RM} \\
\text { control group } \\
1 \mathrm{RM} \text { at baseline, } 6 \text { months, } \\
\text { and } 12 \text { months }\end{array}$ \\
\hline [51] Silva et al. (2016) & $\begin{array}{l}\text { retrospective } \\
\text { cohort study }\end{array}$ & $\begin{array}{c}46 \text { elderly subjects } \\
65-85 \text { yrs } \\
\mathrm{UTr}(n=15) \\
\mathrm{m} \operatorname{Tr}(n=16) \\
\mathrm{i} \operatorname{Tr}(n=15)\end{array}$ & $\begin{array}{l}\text { no acute exercise } \\
\text { regular training for } \\
\geq 5 \text { yrsrunning or ball sports }\end{array}$ & $\begin{array}{c}\mathrm{UTr} \\
\text { never trained } \\
\mathrm{MTr} \\
\text { ball sports or running }<6 \mathrm{~km} \text {, } \\
2-3 \times / \text { week } \\
\mathrm{ITr} \\
\geq 5 \text { days } / \text { week } \\
(>50 \mathrm{~km} / \text { week })\end{array}$ \\
\hline
\end{tabular}

CE: concentric exercise; CG: control group; CON: control; EE: eccentric exercise; F: Friday; IG: intervention group; IGT: impaired glucose tolerance; IST: intensive strength training; ITr: intensely trained; M: Monday; MTr: moderately trained; reps: repetitions; PP: peak power; SET: strength endurance training; UTr: untrained; W: Wednesday; yrs: years; 1RM: one-repetition maximum.

In general, there were no significant differences regarding physical characteristics between the participants reported in all studies. Across all studies, the mean age ranged from 18 to over 75 years. The mean BMI of all participants ranged from 23.21 to $27.9 \mathrm{~kg} \cdot \mathrm{m}^{-2}$. 
Philippe et al. [50] did not provide information about the BMI of participants, but they included no participants with BMI $>30 \mathrm{~kg} \cdot \mathrm{m}^{-2}$. Serostatus of CMV was measured in more than half of the studies $[33,49,52-54]$. Training volume in hours per week retrospectively evaluated or training load measured in different kinds of ways during or before the intervention (e.g., watt or percentage of one-repetition maximum (1RM)) was quantified in all surveys. Heart rate was scaled either as maximal heart rate or as heart rate at rest in three of the studies. Lactate level was only considered in Brown et al. [52]. An overview of examined physical characteristics in each study is shown in the Appendix A (Table A2).

\subsection{Exercise Interventions}

The focus of this review is to examine the effect of PA on the cellular senescence of leukocyte subsets. Therefore, all the included studies investigated PA either via a single bout of exercise, repeated exercise interventions, or via analysis of conducted exercise over the lifespan. Overall, the variance of type of exercise interventions was high. Three of the interventions performed aerobic exercise [49,50,52], including cycling ergometer, treadmill running test, walking exercises, and swimming (see Tables 6 and 7). For the characteristic of intervention performed in the survey of Philippe et al. [50], it is worth mentioning the authors compared concentric uphill walking with eccentric downhill walking. Furthermore, three of the interventions performed resistance exercises between $40 \%$ to $80 \%$ of $1 \mathrm{RM}$. One study compared an aerobic versus a resistance exercise intervention. Two studies did not perform any acute intervention but examined trained subjects, which had different kinds of training levels, with untrained subjects.

Table 7. Summary of studies outcome measures and main results.

\begin{tabular}{|c|c|c|c|c|c|}
\hline Author (Year) & Cohort & $\begin{array}{c}\text { Exercise } \\
\text { Intervention }\end{array}$ & Duration & $\begin{array}{l}\text { Main Outcome } \\
\text { Measures }\end{array}$ & Main Results \\
\hline $\begin{array}{c}\text { [48] Abd } \\
\text { El-Kader et al. } \\
\text { (2018) }\end{array}$ & $\begin{array}{l}\text { sedentary elderly } \\
\text { subjects }(n=60)\end{array}$ & $\begin{array}{l}\text { aerobic endurance } \\
\text { Group A } \\
\text { resistance training } \\
\text { Group B }\end{array}$ & 6 months & $\begin{array}{c}\text { CD3+ } \\
\text { CD4+, and CD8+ } \\
\text { TNF } \alpha, \text { IL-6, and IL-10 }\end{array}$ & $\begin{array}{c}\downarrow \mathrm{TNF} \alpha ; \mathrm{IL}-6(\mathrm{AE}) * \\
\uparrow \mathrm{IL}-10(\mathrm{AE})^{*} \\
\downarrow \mathrm{CD} 3+(\mathrm{AE})^{*} \\
\downarrow \mathrm{CD} 4+; \mathrm{CD} 8+(\mathrm{AE}) * \\
\downarrow \mathrm{CD} 4+/ \mathrm{CD} 8+(\mathrm{AE})^{*}\end{array}$ \\
\hline $\begin{array}{l}\text { [52] Brown et al. } \\
\text { (2014) }\end{array}$ & $\begin{array}{c}\text { male / female } \\
\text { subjects }(n=32) \\
\text { Tr soccer players } \\
\text { UTr }\end{array}$ & $\begin{array}{l}\text { aerobic/anaerobic } \\
\text { endurance } \\
\text { (treadmill running } \\
\text { test to volitional } \\
\text { exhaustion) }\end{array}$ & $\begin{array}{l}\text { single bout of } \\
\text { exercise }\end{array}$ & $\begin{array}{c}\text { CD4+, CD8+, } \\
\text { CD57+, and CD28 } \\
\text { (sen), } \\
\text { CD57, CD28+ (naïve) }\end{array}$ & $\begin{array}{c}\downarrow \text { CD4+ sen }(\mathrm{Tr}){ }^{*} \\
\uparrow \mathrm{CD} 4+\text { naïve }(\mathrm{Tr}) \\
\downarrow \text { CD8+ sen }(\mathrm{Tr}){ }^{*} \\
\uparrow \mathrm{CD} 8+\text { naïve }(\mathrm{Tr}){ }^{*} \\
\uparrow \mathrm{CD} 4+\text { sen, CD8+ sen }(\mathrm{m}) * \\
\downarrow \text { CD4+ naïve, CD8+ naïve }(\mathrm{m}) *\end{array}$ \\
\hline $\begin{array}{l}\text { [33] Dinh et al. } \\
\text { (2019) }\end{array}$ & $\begin{array}{l}\text { elderly women } \\
\qquad(n=100)\end{array}$ & $\begin{array}{c}\text { resistance training } \\
\text { IST, SET } \\
\text { stretching } \\
\text { CON }\end{array}$ & 6 weeks & $\begin{array}{c}\mathrm{CD}^{+} \\
\mathrm{CD} 8^{-} \\
\mathrm{CD} 28, \text { and CD57 }\end{array}$ & 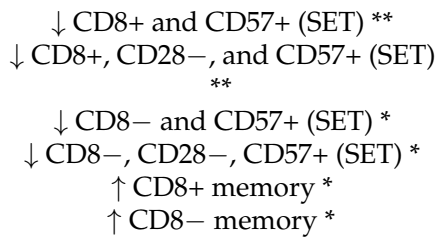 \\
\hline $\begin{array}{l}\text { [54] Moro-García } \\
\text { et al. (2014) }\end{array}$ & $\begin{array}{l}\text { athletes/non- } \\
\text { athletes } \\
\quad(n=95)\end{array}$ & $\begin{array}{c}\text { no intervention } \\
\text { athletes performing } \\
\text { aerobic/anaerobic } \\
\text { endurance, } \\
\text { resistance training }\end{array}$ & - & $\begin{array}{c}\mathrm{VO}_{2 \max } \\
\text { physical activity } \\
\text { score } \\
\text { TREC content } \\
\text { CD4+, CD8+, CD16+, } \\
\text { and CD56+ } \\
\text { CD19+ and CD69 }\end{array}$ & $\begin{array}{c} \\
\downarrow \text { CD4+ (A)* } \\
\uparrow \mathrm{CD} 8+(\mathrm{A}) * \\
\uparrow \text { NK cells (A) ** } \\
\downarrow \text { TREC } / \text { CD8+ (YA) * } \\
\downarrow \text { Neutrophils, Lymphocytes (A)* }\end{array}$ \\
\hline
\end{tabular}


Table 7. Cont.

\begin{tabular}{|c|c|c|c|c|c|}
\hline Author (Year) & Cohort & $\begin{array}{c}\text { Exercise } \\
\text { Intervention }\end{array}$ & Duration & $\begin{array}{l}\text { Main Outcome } \\
\text { Measures }\end{array}$ & Main Results \\
\hline $\begin{array}{l}\text { [47] Neves et al. } \\
\text { (2009) }\end{array}$ & $\begin{array}{l}\text { elderly women } \\
\qquad(n=15)\end{array}$ & $\begin{array}{c}\text { resistance exercise } \\
(\mathrm{S} 50 \%, \mathrm{~S} 80 \%, \mathrm{SC})\end{array}$ & acute exercise & $\begin{array}{c}\text { LT } \\
\text { CT, IgA } \\
\text { Total Lymphocytes } \\
\text { CD4+, CD8+ }\end{array}$ & $\begin{array}{c}\downarrow \mathrm{CT}(\mathrm{T} 3 ; \mathrm{S} 50 \%, \mathrm{~S} 80 \%, \mathrm{SC}) * \\
\uparrow \mathrm{LT}(\mathrm{T} 2 ; \mathrm{SC}) * \\
\uparrow \mathrm{IgA}(\mathrm{T} 2 \mathrm{~S} 50 \% ; \mathrm{T} 2, \mathrm{~T} 3 \mathrm{~S} 80 \%) * \\
\uparrow \text { Total Lymphocytes (T3) * } \\
\uparrow \mathrm{CD} 4+(\mathrm{T} 3, \mathrm{~S} 80 \%, \mathrm{SC}) * \\
\uparrow \mathrm{CD} 8+(\mathrm{T} 3, \mathrm{SC}) *\end{array}$ \\
\hline $\begin{array}{l}\text { [50] Philippe } \\
\text { et al. (2019) }\end{array}$ & $\begin{array}{l}\text { older adults } \\
\qquad(n=16)\end{array}$ & $\begin{array}{l}\text { aerobic endurance } \\
\text { (walking exercise) }\end{array}$ & 3 weeks & $\begin{array}{c}\text { TNF } \alpha, \text { IL-6 } \\
\text { CD3+, CD4+, CD8+ } \\
\text { CD45RO, CCR7 } \\
\text { CD16+, CD19+ } \\
\text { CD25+ }\end{array}$ & $\begin{array}{c}\uparrow \mathrm{CD} 4+/ \mathrm{CD}^{+*} \\
\uparrow \mathrm{CD} 4+/ \mathrm{CD}^{+*} \\
\downarrow \mathrm{CD} 8+/ \mathrm{CD}^{+*} \\
\uparrow \mathrm{CD} 4+/ \mathrm{CCR} 7+/ \mathrm{CD}^{+} 5 \mathrm{RO}^{+*} \\
\downarrow \mathrm{CD}_{+}+/ \mathrm{CCR} 7-/ \mathrm{CD} 4 \mathrm{RO}^{-*} \\
\downarrow \mathrm{CD} 16^{+*} \\
\uparrow \mathrm{CD} 8+/ \mathrm{CCR}+/ \mathrm{CD} 45 \mathrm{RO}+* \\
\uparrow \mathrm{CD} 8+/ \mathrm{CCR} 7+/ \mathrm{CD} 45 \mathrm{RO}-* \\
\downarrow \mathrm{CD} 8+/ \mathrm{CCR} 7-/ \mathrm{CD} 45 \mathrm{RO}-*\end{array}$ \\
\hline $\begin{array}{l}\text { [49] Pistillo et al. } \\
\text { (2013) }\end{array}$ & $\begin{array}{l}\text { active males } \\
\quad(n=34)\end{array}$ & $\begin{array}{l}\text { aerobic endurance } \\
\text { (cycle ergometer) }\end{array}$ & $\begin{array}{l}\text { single bout of } \\
\text { exercise }\end{array}$ & $\begin{array}{c}\text { viral serostatus } \\
\text { Total Lymphocytes } \\
\text { CD3+ and } \gamma \delta \text { T-cells } \\
\text { KLRG1 }\end{array}$ & $\begin{array}{c}\downarrow \gamma \delta \text { T-cells (old) } \\
\uparrow * * * \\
\gamma \delta \text { T-cells } \mathrm{CMV}^{+} \text {(young) } \\
\uparrow * * \\
\uparrow \delta \text { T-cells (post exercise) } \\
\uparrow \text { KLRG1+ } \gamma \delta \text { T-cells (young) }\end{array}$ \\
\hline $\begin{array}{l}\text { [53] Raso et al. } \\
\text { (2007) }\end{array}$ & $\begin{array}{l}\text { elderly sedentary } \\
\text { women }\end{array}$ & $\begin{array}{l}\text { resistance exercise } \\
\text { (light to moderate, } \\
\text { IG) } \\
\text { no exercise } \\
\text { CG }\end{array}$ & 12 months & $\begin{array}{c}\text { CD4+, CD8+ } \\
\text { CD45RA+, and } \\
\text { CD45RO+ } \\
\text { NKCA } \\
\text { CD56 }{ }^{\text {dim }} \text { and } \\
\text { CD56 } 6^{\text {bright }} \\
\text { Total Lymphocytes }\end{array}$ & $\begin{array}{c}\uparrow \mathrm{CD} 4+/ \mathrm{CD} 45 \mathrm{RA}+(\mathrm{IG}) \\
\uparrow \mathrm{CD} 8+/ \mathrm{CD} 45 \mathrm{RA}+(\mathrm{IG}) \\
\downarrow \mathrm{CD} 4+/ \mathrm{CD} 45 \mathrm{RO}+(\mathrm{IG}) \\
\downarrow \mathrm{CD} 8+/ \mathrm{CD} 45 \mathrm{RO}+(\mathrm{IG}) \\
\uparrow \mathrm{CD} 56^{\mathrm{dim}} / \mathrm{CD} 56^{\mathrm{bright}} \\
\text { (no significant differences) }\end{array}$ \\
\hline $\begin{array}{l}\text { [51] Silva et al. } \\
\text { (2016) }\end{array}$ & $\begin{array}{l}\text { elderly subjects } \\
\qquad(n=46) \\
\text { UTr, MTr, ITr }\end{array}$ & $\begin{array}{c}\text { no intervention } \\
\text { aerobic endurance } \\
\text { ball sports }\end{array}$ & $\geq 5$ years & $\begin{array}{l}\text { Questionnaires } \\
\text { (e.g., SF-36) } \\
\text { CD3+, CD4+, and } \\
\text { CD8+ } \\
\text { CD45RO and CD28 } \\
\text { TL (CD4+, CD8+) }\end{array}$ & $\begin{array}{c}\downarrow \text { CD8+ TEMRA (MTr, ITr) } \\
\downarrow \text { CD4+ TEMRA (ITr) }{ }^{* *} \\
\uparrow \text { CD4+ CM (MTr) }{ }^{*} \\
\uparrow \text { T-cell TL (MTr, ITr })^{*} \\
\uparrow \text { CD8+ EM (ITr) * } \\
1 / 3(\text { CD4+ TEMRA/CD8+ TEMRA) }\end{array}$ \\
\hline
\end{tabular}

${ }^{*} p<0.05 ;{ }^{* *} p<0.01 ;{ }^{* * *} p<0.001 ; \uparrow:$ increase; $\downarrow$ : decrease; $1 / 3$ : ratio; A: athletes; AE: aerobic endurance; CG: control group; CM: central memory T-cells; CMV+: cytomegalovirus positive; CON: control; CT: cortisol; EM: effector memory T-cells; IG: intervention group; IgA: immunoglobulin A; IST: intensive strength training; ITr: intensely trained; m: males; LT: total leukocytes; MTr: moderately trained; NK cells: natural killer cells; NKCA: natural killer cell activity; SC: no exercise control session; sen: senescent; SET: strength endurance training; SF-36: Short Form (36) Health Survey; S50\%: Session 50\% 1RM; S80\%: Session 80\% 1RM; TEMRA: T effector memory cells re-expressing CD45RA; Tr: trained; TREC content: T-cell receptor excision circle content; TL: telomere length; T2: directly after exercise; T3: 3 h post exercise; UTr: untrained; $\mathrm{VO}_{2 \max }$ : maximal oxygen uptake; YA: young athletes; $1 \mathrm{RM}$ : one-repetition maximum.

Trained subjects performed soccer or other ball sports, rowing, running, or resistance training. Sedentary subjects did not perform exercise training, depending on the survey, over their lifetime, for the past 6 months, not more than 2-3 times per week, or not more than $150 \mathrm{~min}$ per week. The duration of the exercise performed during the survey varies between one single bout of exercise, some weeks, 6 months, or up to a whole year. The exercise interventions were mainly supervised or monitored by the authors or at least one of them since they took place in rehabilitation centers or in research facilities. The three studies which conducted acute exercise interventions assessed the exercise performance capacity of the participants with a treadmill running test to volitional exhaustion [52], a cycling ergometer test at $80-85 \%$ peak power [49], or sessions of resistance exercise at $50 \%$ or $80 \% 1 \mathrm{RM}$ [47].

\subsection{Assessment of Leukocyte Subpopulations}

Studies were assessed for the process of taking blood samples and analyzing these samples. The focus was set on leukocyte subtypes and their quantification. In all studies, blood samples were taken from the participants at different time intervals and stages. A detailed overview of the blood collection procedure in chronological order with the exercise intervention is depicted in Figure 3. Monoclonal antibodies for phenotypic markers 
were used to quantify different kinds of leukocyte subsets. Blood samples were mixed with ethylenediaminetetraacetic acid (EDTA) to prevent coagulation in four of the studies $[33,47,53,54]$. The other five studies did not provide any information about the usage of anticoagulants. Even though it was the aim to include all kinds of leukocytes in this review, mainly T-cells, especially CD4+ and CD8+, were investigated. Overall, monoclonal antibodies were conjugated with fluorescein isothiocyanate (FITC), phycoerythrin (PE), phycoerythrin-cyanines (PE-Cy5 and PE-Cy7), allophycocyanins (APC and APC-Cy7), peridinin chlorophyll proteins (PerCP and PerCP Cy-5.5), Alexa flour (Alexa488), V500, or V450 to stain different leukocyte subsets. Stained cells were quantified in each analysis using flow cytometry.

[48] Abdl El-Kader et al. (2018)

[52] Brown et al. (2014)

[33] Dinh et al. (2019)

[54] Moro-García et al. (2014)

[47] Neves et al. (2009)

[50] Philippe et al. (2019)

[49] Pistillo et al. (2013)

[53] Raso et al. (2007)

[51] Silva et al. (2016)

\begin{tabular}{|c|c|c|c|c|c|c|}
\hline & pre & 6 months & post & \multirow[b]{2}{*}{ 1h-post } & \\
\hline & & pre & acute exercise & post & & \\
\hline & & \multirow[t]{2}{*}{ pre } & 6 weeks & 24-48h post & & \\
\hline & & & no acute exercise & $>18 \mathrm{~h}$ last PA & & \\
\hline & & pre & acute exercise & post & 3h-post & $48 \mathrm{~h}$ after pre \\
\hline & & pre & 3 weeks & $1-2 d$ post & & \\
\hline & pre $1-2 w$ & pre & acute exercise & post & 1h-post & \\
\hline pre & 6 months & post & further 6 months & $12 \mathrm{~m}$-post & & \\
\hline & & & no acute exercise & $\geq 48 \mathrm{~h}$ last PA & & \\
\hline
\end{tabular}

Figure 3. Scheme of time when blood samples were taken in connection with the time exercise intervention took place. Blood samples were taken pre-intervention and post-intervention, highlighted in grey. Moro-García et al. [54] and Silva et al. [51] investigated retrospectively and therefore only took one blood sample. In the other studies, there was at least one examination pre-exercise and post-exercise. Training periods are marked in red and highlighted in white. d: day(s); h: hour(s); m: months; PA: physical activity; w: week(s).

Investigated leukocyte cell subsets and results of interventions are presented in Table 7 for each study. CD4+ and CD8+ T-cells were examined in all studies, except Dinh et al. [33], who only investigated CD8+ and CD8-, and Pistillo et al. [49], who focused on $\gamma \delta$ Tcells. The authors of described surveys considered different subsets of naïve, senescent, and memory T-cells. In some studies, cytokines and inflammation markers were also measured. Mainly results were presented as a percentage of $\mathrm{CD} 3+$ or as total numbers (cell counts). Neves et al. [47] described total lymphocytes, CD4+, CD8+, plus IgA and cortisol levels. Abd El-Kader et al. [48] quantified CD3+, CD4+, and CD8+ T-cells as well as the CD4+/CD8+ ratio, IL-6, IL-10, and TNF- $\alpha$. In Brown et al. [52], the percentage of total $\mathrm{CD} 3+$ and of senescent and naïve CD4+ and CD8+ T-cells were presented. T-cells expressing CD28-/CD57+ (senescent phenotype) and CD28+/CD57- (naïve phenotype) were quantified. Dinh et al. [33] proceeded similar and separated CD8+ and CD8- T-cells into four distinct subpopulations: CD28+/CD57 - (naïve), CD28-/CD57(memory) and CD28-/CD57+ or CD28+/CD57+ (senescence-prone). Pistillo et al. [49] classified the percentage of $\gamma \delta$ T-cells in the CD3+ cell population. Then they determined KLRG1+ cells among those $\gamma \delta$ T-cells, along with CD3+ $/ \gamma \delta$ TCR+/CD4-/CD8-. Besides analysing the $\mathrm{CD} 4+/ \mathrm{CD} 8+$ ratio, Philippe et al. [50] acknowledged the CD4+/CD3+ and $\mathrm{CD} 8+/ \mathrm{CD} 3+$ ratio. Cell type was specified using the percentage of all lymphocytes. Comprised cell types were combinations of CD4+ or CD8+ with CCR7 and CD45RO. The authors differed between naïve T-cells (CD45RO-/CCR7+), CM (CD45RO+/CCR7+), EM $(\mathrm{CD} 45 \mathrm{RO}+/ \mathrm{CCR7}-)$ and TEMRA (CD45RO-/CCR7-) cells. CD16+ (NK cells, $\gamma \delta \mathrm{T}$-cells, and subsets of CD8+), CD19+ (B-lymphocytes), cell surface marker CD25+ for CD4+ and CD8+ plus TNF- $\alpha$ and IL- 6 were also analysed. Raso et al. [53] specified NK cell activity (NKCA) and examined NK cell subsets (CD56 ${ }^{\mathrm{dim}}$ and CD56 ${ }^{\text {bright }}$ ). They included naïve $(\mathrm{CD} 45 \mathrm{RA}+)$ and memory $(\mathrm{CD} 45 \mathrm{RO}+)$ subsets of $\mathrm{CD} 4+$ and CD8+ T-cells as well. In addition, $\mathrm{CD} 19+, \mathrm{CD} 25+$ (activation marker), CD28+ (co-stimulatory molecule), CD69+ (activation marker) and CD95+ (apoptotic marker) were brought into focus. Moreover, Silva et al. [51] 
compared the frequency of different combinations of CD4+ and CD8+ with CD45RA/CCR7: naïve (CD45RA+/CCR7+), CM (CD45RA-/CCR7+), EM (CD45RA-/CCR7-) and TEMRA (CD45RA+/CCR7-) T-cells. Secondary, telomere length of CD4+ and CD8+ T-cell subsets expressing CD45RO and CD28 was analysed. In these subpopulations, the telomere peptide nucleic acid (PNA) was used to calculate telomere length [51].

Furthermore, the expression of caspase-3 and B-cell lymphoma 2 (Bcl-2) in T-cell subsets and cytokine frequency (e.g., TNF $\alpha$ and IFN $\gamma$ and IL-6 and IL-10) were quantified [51]. In the survey of Moro-García et al. [54], the authors analyzed the T-cell receptor excision circle (TREC) for CD4+ and CD8+. Overall, the authors compared the percentage of CD4+, CD8+, CD16+, CD56+, and CD19+ between the intervention groups. For CD4+ and CD8+, they also investigated naïve, CM, EM, and TEMRA T-cell subsets via CD45RA and CCR7. Additionally, the composition of naïve, EM, and TEMRA cells was examined by determining the frequency of CD31 to monitor recent thymic emigration. Besides, the functional ability of NK cells by expression of CD69+ and CD107a and the proliferative capacity of CD4+ and CD8+ were evaluated.

\subsection{Effects of Physical Activity on Cellular Senescence and Leukocyte Subpopulations}

The main outcome measures and main results are shown in Table 7 . Results were accepted as significant for $p<0.05$ in all studies. Depending on study design, normal distribution was assessed using the Kolmogorov-Smirnov test. Nonparametric tests like the Mann-Whitney U-Test or Wilcoxon rank-sum test were used for non-normal distributed data. Associations were explored with Spearman's rank correlation test, while $t$-tests and analysis of variance or covariance (ANOVA and ANCOVA) were also used as statistical tools depending on the data set. Following this, the results are sorted according to the conducted exercise intervention. First, the studies not including any acute intervention but comparing trained with untrained subjects are described. Secondly, studies investigating endurance exercises (aerobic and anaerobic) are presented. Then, studies examining resistance programs are shown. At last, one study comparing endurance and resistance training is specified.

\subsubsection{Trained vs. Untrained Subjects without Exercise Intervention}

Silva et al. [51] did not perform an acute exercise intervention; however, they investigated three different groups of elderly participants with either an intense training lifestyle (ITr), a moderate training lifestyle (MTr), or untrained subjects (UTr). The three groups showed a contraction of the CD4+/CD8+ ratio; the average was 2 . There was a decrease in naïve T-cells and implicated an increase in memory T-cells. Concerning CD4+ T-cells, there was a twofold lower proportion of TEMRA cells in the training groups compared to UTr (UTr vs. MTr, $p<0.001$; UTr vs. ITr, $p<0.01$ ). The proportion of CD8+ TEMRA cells was also lower in training groups (significant for UTr vs. ITr, $p<0.01$ ). Furthermore, there was a threefold higher amount of CD8+ TEMRA cells compared with CD4+ TEMRA cells. The ITr group had a higher amount of CD8+ EM cells than the other groups $(p<0.05)$, whereas the MTr group was associated with a higher proportion of CD4+ CM cells $(p<0.05)$ in comparison with ITr and UTr. Both training groups showed no significant modification of the amount of senescent CD8+/CD28- T-cells. However, telomeres of T-cells were significantly longer. In the ITr group, telomere length increased mainly in CD8+ T-cells $(p<0.05)$, whilst a moderate increase was observed for CD8+ and CD4+ T-cells in MTr. The determination of the expression of proapoptotic molecule caspase- 3 on CD4+/CD45RO+ and CD4+/CD45RO- showed that almost no apoptosis occurred in the CD4+ T-cells for the ITr group compared to UTr $(p<0.001)$. Consistent with this result, anti-apoptotic $\mathrm{Bcl}-2$ expression was higher in ITr than in the other groups $(\mathrm{CD} 4+/ \mathrm{CD} 45 \mathrm{RO}+, p<0.01$; CD4+/CD45RO-,$p<0.001)$.

Conversely, Moro-García et al. [54] found no differences in and differentiation state of CD8+ T-cells due to activity level; they only observed an influence of age in CMV+ subjects in naïve $(p<0.001)$ and TEMRA $(p<0.01)$ subsets. In young CMV - athletes, 
the authors found a decrease in naïve $(p<0.01)$ and CM cells $(p<0.05)$ regarding CD4+ T-cell differentiation, and an increase in CD4+ TEMRA cells $(p<0.01)$. In general, high volume exercise generated a decrease in percentage of CD4+ T-cells for CMV+ subjects $(p<0.05)$ and an increase in CD8+ T-cells $(p<0.05)$ and NK cells $(p<0.01)$ compared with non-athletes. There was no significant difference in PA level and proportion of CD19+. The TREC content was lower in CD8+ cells for young athletes in both CMV+ and CMV - individuals $(p<0.05)$, and they as well had lower frequencies of recent naïve CD8+ cells thymic emigration $(\mathrm{CD} 31+, p<0.05)$. Functional ability of CD4+ and CD8+ T-cells was impaired in young athletes. However, NK-cells showed a higher activation and degranulation.

\subsubsection{Endurance Exercise Intervention}

Unlike the above-described studies without acute PA, Brown et al. [52] examined the effect of a single bout of maximal endurance exercise on senescent T-cells. Senescent CD8+ T-cells increased immediately post-exercise, though decreased $1 \mathrm{~h}$ post-exercise $(p<0.05)$. For the senescent CD4+ T-cells, a similar pattern was observed; however, only the decrease from post-exercise to $1 \mathrm{~h}$ post-exercise was significant $(p<0.05)$. Percentage of naïve CD8+ T-cells decreased immediately post-exercise and increased $1 \mathrm{~h}$ post-exercise $(p<0.05)$. For naïve CD4+ T-cells a similar response was observed. Likewise, only the post-exercise to $1 \mathrm{~h}$ post-exercise period increase was significant $(p<0.05)$. Training status affected senescent CD4+ and CD8+ T-cells, whereby senescent cell subsets were higher in UTr vs. trained $(\operatorname{Tr})(p<0.05)$. Percentage of naïve CD8+ T-cells was lower in UTr vs. Tr subjects $(p<0.05)$. Regarding the effect of sex on senescent CD4+ and CD8+ T-cells, the proportion of senescent subsets was higher in males vs. females. For naïve CD4+ and CD8+ T-cells, the overall proportion was lower in males vs. females. Especially UTr males had a higher percentage of senescent and a lower percentage of naïve CD4+ T-cells $(p<0.01)$.

Philippe et al. [50] observed a significant increase in the CD4+/CD8+ and CD4+/CD3+ ratio after 3 weeks of eccentric or concentric walking exercise $(p<0.05)$, as well as a decline of the CD8+/CD3+ ratio $(p<0.05)$. Furthermore, there was a rise of proportion of CD4+ CM cells $(p<0.05)$ and a reduction in CD4+ TEMRA cells and CD16+ cells found after training $(p<0.05)$. An increase was discovered for CD8+ CM cells $(p<0.01)$ and naïve CD8+ T-cells $(p<0.05)$, while CD8+ TEMRA cells decreased $(p<0.05)$. ANCOVA showed no significant group effect between the eccentric and concentric walking group. There was neither a correlation between serostatus of CMV and any T-cell subpopulation before exercise nor after intervention-induced changes in subsets.

As described, Pistillo et al. [49] used a different approach to this topic by investigating $\gamma \delta$ T-cells. Young and elderly males, matched for CMV, EBV, HSV-1, and parvovirus B19 (PVB19), conducted a single bout of cycling exercise. Generally, the percentage of $\gamma \delta$ T-cells was significantly lower in older compared to younger subjects $(p<0.001)$. CMV serology did not affect $\gamma \delta$ T-cells proportions in older subjects; however, there were greater numbers of $\gamma \delta$ T-cells in young CMV+ subjects compared to non-infected young counterparts $(p<0.01)$. Immediately after exercise, total numbers of $\gamma \delta$ T-cells, as well as KLRG1+ and KLRG1- subsets, were greater than pre and $1 \mathrm{~h}$ post-exercise $(p<0.01)$. Young athletes redeployed greater numbers of $\gamma \delta$ T-cells and KLRG1 $+\gamma \delta$ T-cells than the older athletes $(p<0.01)$. ANOVA showed that the interaction effect of age and exercise was due to a greater mobilization of absolute $\gamma \delta$ T-cell numbers $(p<0.001)$. EBV, HSV-1, and PVB19 serology was not associated with $\gamma \delta$ T-cell appearance.

\subsubsection{Resistance Exercise Intervention}

Neves et al. [47] examined the effects of either a resistance exercise session at $50 \%$ $1 \mathrm{RM}(\mathrm{S} 50 \%), 80 \%$ 1RM (S80\%), or a control session (SC) without exercise on leukocyte counts and cortisol plus IgA levels of elderly women. All sessions resulted in a significant reduction in cortisol levels $3 \mathrm{~h}$ post-exercise (T3) compared to baseline (T1) $(p<0.05)$. IgA was elevated immediately after exercise sessions (T2) but not after SC $(p<0.05)$. For the 
session performed at an intensity of $80 \%$ of $1 \mathrm{RM}(\mathrm{S} 80 \%)$, this elevation remained until T3. Total leukocytes were only elevated at T2 in relation to T1 $(p<0.05)$. Three hours post-exercise (T3), total lymphocyte count was elevated significantly in all three groups. CD4+ T-cells were elevated at T3 for the S80\% and SC session $(p<0.05)$, but not for the S50\% session. For CD8+ T-cells, there was only a significant elevation after the SC session at T3 $(p<0.05)$.

Similar to these findings, Raso et al. [53] also could not provide explicit results. The authors investigated immunological parameters of sedentary elderly women before, after 6 months, and after 12 months of either resistance training or a control group. There were no significant exercise-induced changes in total lymphocyte count or subsets, as well as no significant differences between the two groups at any measuring point. In general, CD4+ and CD8+ T-cells comprised more memory than naïve cell subsets at baseline for both groups. There was a non-significant tendency in the intervention group for an increase in CD4+ and CD8+ naïve T-cells and a decrease in memory T-cells. The NK cell marker CD56dim was predominated in both groups, as expected for healthy subjects, but demonstrated no effect of the exercise intervention. The observation of CD19, CD25, CD28, CD69, CD95, and NKCA did not show any detectable effect for both groups, too.

On the other hand, Dinh et al. [33] showed clearer results after 6 weeks of strengthendurance training ( $40 \% 1 \mathrm{RM}$, SET) compared with intensive strength training (80\% 1RM, IST), and a control group (stretching, CON). There was a significant decrease in the proportion of senescent CD8+/CD57+ cells $(p<0.01)$, especially the CD8+/CD28-/CD57+ subset $(p<0.01)$ overall and within groups for the SET group $(p<0.01)$. Regarding the CD8- T-cell subset, there was a trend towards a decrease in senescent CD8-/CD57+ cells overall, but only significantly in the SET group $(p<0.05)$. This effect is mostly caused by the decrease in CD8 - /CD28-/CD57+ in the SET group $(p<0.05)$. The percentage of memory subtype of CD8+ and CD8 - showed an overall increase $(p<0.05)$. However, no statistically significant changes were observed within the different exercise groups. For naïve CD8+ and CD8subpopulations, there was no difference observed after 6 weeks. Comparing absolute numbers of CD8 cells, within-group analysis disclosed an exercise-induced decrease in the count of all phenotypes for CD8+ subsets in the SET group $(p<0.05)$. There was a traininginduced reduction in the CD8+/CD57+ peripheral cell count and CD8+/CD28-/CD57+ for the SET group compared to CON $(p<0.05)$. For absolute numbers in CD8- T-cell subsets, as well as for naïve and memory T-cells, there was no statistically significant difference found.

\subsubsection{Aerobic Endurance vs. Resistance Exercise Intervention}

The study of Abd El-Kader et al. [48] investigated aerobic endurance exercise and resistance exercise. Sedentary elderly subjects performed either aerobic training or a resistance training program for 6 months. Numbers of CD3+, CD4+, and CD8+ cells decreased significantly in the aerobic exercise (AE) group after 6 months $(p<0.05)$ and enhanced in the resistance exercise (RE) group (not significantly). The CD4+/CD8+ ratio also declined in the AE group $(p<0.05)$ and increased in the RE group. For inflammatory cytokines, TNF- $\alpha$ and IL- 6 decreased, whereas IL-10 increased significantly in the AE group $(p<0.05)$. Additionally, there were significant differences between the mean levels of investigated cell counts overall in the AE and RE group after intervention $(p<0.05)$.

\subsection{Risk of Bias}

The risk of bias was assessed, as explained in the methods section, to obtain an overview of study quality (see 3.2 Study quality assessment). Only adequately randomized and controlled studies were accepted as RCTs. Three studies were identified as RCTs and were assessed with the RoB2 Tool [44]. The other studies did not fulfill the criteria for RCTs and had to be assessed using the ROBINS-I tool to evaluate non-randomized or non-controlled studies adequately for the effects of interventions [45]. Studies with two intervention groups and one control group were classified as non-RCTs. Studies comparing 
two intervention groups without a control group were categorized as non-controlled trials. Observational studies, such as retrospective cohort studies, were also included. Therefore, six studies were analyzed with the ROBINS-I Tool. The summary of the results for nonRCTs, non-controlled trials, and observational studies assessed with the ROBINS-I Tool is shown in Table 8. The studies are sorted alphabetically.

Table 8. Risk of bias assessment with the ROBINS-I tool for the included studies $(n=6)$.

\begin{tabular}{|c|c|c|c|c|c|c|c|c|}
\hline Author & Confounding & $\begin{array}{c}\text { Selection } \\
\text { Participants }\end{array}$ & $\begin{array}{l}\text { Classification } \\
\text { Intervention }\end{array}$ & $\begin{array}{c}\text { Deviation } \\
\text { Intended } \\
\text { Intervention }\end{array}$ & $\begin{array}{l}\text { Missing } \\
\text { Data }\end{array}$ & $\begin{array}{c}\text { Outcome } \\
\text { Measurement }\end{array}$ & $\begin{array}{c}\text { Selection } \\
\text { Reported } \\
\text { Results }\end{array}$ & $\varnothing$ \\
\hline [48] Abd El-Kader et al. & + & + & + & \pm & + & \pm & + & \pm \\
\hline [52] Brown et al. & + & + & + & + & + & \pm & \pm & \pm \\
\hline [54] Moro-García et al. & \pm & \pm & + & + & + & \pm & + & \pm \\
\hline [50] Philippe et al. & + & + & + & \pm & $?$ & \pm & + & \pm \\
\hline [49] Pistillo et al. & \pm & + & \pm & $?$ & $?$ & \pm & + & $\times$ \\
\hline [51] Silva et al. & \pm & \pm & + & + & + & \pm & + & \pm \\
\hline
\end{tabular}

+: low risk of bias; \pm : moderate risk; $\times$ : serious risk; ?: unclear risk.

All studies assessed with the ROBINS-I tool showed some concerns regarding the risk of bias. Especially the domains 'confounding' and 'outcome measurement' were somehow risky for most of the studies. None of the studies blinded outcome assessors nor participants. So, this domain showed a moderate risk for all seven interventions. The domain 'missing data' was considered as at low or unclear risk in the analyzed studies since usually all participants could be investigated, or it was not approached that data was missing during the investigation. Therefore, it was supposed that no data was missing. Pistillo et al. [49] indicated an overall serious risk of bias. They described the two domains deviation to intended intervention and missing data not sufficiently. Hence, their study was considered as having a serious risk of bias. Abd El-Kader et al. [48] did not provide any information about the sex of the participants; however, they assigned them in a randomized manner to the intervention groups. Silva et al. [51] did not consider the sex of the subjects as a possible confounder.

The three analyzed studies using the RoB2 Tool (presented in Table 9) were all at low risk of bias for randomization and outcome measurements but showed some concerns regarding the assignment.

Table 9. Studies assessed using the RoB2 Tool $(n=3)$.

\begin{tabular}{cccccc}
\hline Author & Randomization & Assignment & Missing Data & $\begin{array}{c}\text { Outcome } \\
\text { Measurment }\end{array}$ & $\begin{array}{c}\text { Selection } \\
\text { Reported Results }\end{array}$ \\
\hline [33] Dinh et al. & + & \pm & + & \pm & + \\
\hline [47] Neves et al. & + & \pm & - & + & + \\
\hline [53] Raso et al. & + & \pm & + & + \\
\hline
\end{tabular}

In all three studies, it was not sufficiently described how the authors assigned the participants into the intervention or control group. Although there were some concerns due to the domain 'assignment', the study from Raso et al. [53] was rated as low risk of bias because there were no other concerns than in this domain. Neves et al. [47] did not provide clear information on the data for most of the randomized participants. They did not adequately and unambiguously explain the process of data survey. Thus, it was unclear how they assigned the participants to the intervention groups. They also did not address missing data at all. Therefore, this investigation process and the study results were evaluated as at 'high risk'. 


\section{Discussion}

Physical activity (PA) has many positive health benefits, such as higher levels of cardiorespiratory and muscular fitness, lower body fat mass, enhanced bone health, higher levels of functional health, better cognitive function, lower rates of all-cause mortality, and prevention of various diseases [55]. However, there is still a lack of high-quality studies and systematic reviews examining the effects of different kinds of PA on the aging immune system, which can contribute to the understanding of cellular senescence and how to delay far-reaching impacts on the immune function. Hence, the aim of this systematic review was to precisely investigate the effects of PA on the aging immune system in general and the cellular senescence of leukocyte subsets. PA was defined as endurance or resistance exercise training. Thereby, it was possible to compare the effects of different types of exercise interventions to potentially determine which exercise protocol provides the most beneficial consequences in relation to cellular senescence.

Within this review, a total of nine studies were included after conducting systematic literature research using PubMed and Web of Science as databases. The included studies were analyzed regarding their cohorts, exercise interventions, examined leukocyte subsets and their results. The focus of this review was to select comparable studies and a similar approach to investigate this research field, which has been achieved partly because of heterogenic study designs. Exercise interventions differed regarding the duration, intensity, composition, and impact on metabolism. Examined cell subsets varied as well. However, mainly T-cells, especially the CD4+ and CD8+ subtypes, were considered. For CD4+ and $\mathrm{CD} 8+$, particularly the naïve, effector memory (EM), central memory (CM), and EM cells that re-express CD45RA (TEMRA) subsets were brought into focus. Active groups were compared to sedentary controls, to baseline values, or to active controls. Additionally, studies focused on either a single bout of exercise, an exercise program of some weeks or months, or retrospectively on impacts of long-term exercise and lifelong PA. However, there were also some studies conducting endurance exercise and resistance exercise. So, a comparison of these training protocols was possible.

Silva et al. [51] showed a shift from predominantly naïve T-cells to predominantly memory T-cell proportions in the elderly, which reflects the influence of exposure to antigens over the lifespan. Moderate and intense aerobic training seems to attenuate some of the effects of aging on memory and TEMRA T-cells. This may demonstrate a better immune response in trained elderly subjects. Moderate training lifestyle was associated with a decreased percentage of particularly CD4+ TEMRA cells, whereas an intense training lifestyle was associated with decreased percentage of CD4+ and CD8+ TEMRA cells [51]. On the contrary, Moro-García et al. [54] found opposite results referring to TEMRA subsets. Elderly athletes had significantly higher levels of CD4+ TEMRA cells, as well as slightly increased CD8+ TEMRA proportions, compared with non-athletes. Active individuals, in general, had a decrease in CD4+ and an increase in CD8+ and NK cells. Hence, athletes had an inverted CD4+/CD8+ ratio [54]. This matches with the general assumption that aging is associated with a reduction in the $\mathrm{CD} 4+/ \mathrm{CD} 8+$ ratio [56]. A positive change in Th1 to Th2 helper cells ratio has also been suggested as an effective response to PA [57]. Though, Silva et al. [51] found only a few trained and untrained subjects with a decreased $\mathrm{CD} 4+/ \mathrm{CD} 8+$ ratio. In conclusion, considering long-term exercise over the lifespan, there were inconsistent findings regarding the effect of PA on the CD4+/CD8+ ratio for the two studies included in this review. A possible explanation for this could be the differences in exercise interventions, since Moro-García et al. [54] examined athletes who participated in aerobic and anaerobic endurance, resistance exercise training, stretching, and body-core exercises, whereas Silva et al. [51] mainly investigated endurance-trained subjects, who were runners. This points to the assumption of Silva et al. [51] that an extensive mix of aerobic and anaerobic exercise has a different impact on the immune system than regular aerobic exercise. Supportive, Saygin et al. [58] found changes in immune parameters dependent on the type of PA by investigating sedentary, moderately active, and highly 
active athletes. Moderate, regular exercise can potentially affect the protective antibody response and leukocyte proportions more positively [58].

The reduced adaptive immune response in young athletes might be the result of increased cytotoxicity of NK cells, which may affect the efficacy of the immune response [54]. A possible explanation for this is that NK cell cytotoxicity could be a compensatory mechanism to maintain or even improve adequate immunity [54], which was also suggested in another study by McFarlin et al. [59], who examined NK cell activity before and after 10 weeks of regular resistance exercise.

Accordingly to the findings of Moro-García et al. [54] for athletes with higher impact exercise, resembling results were published by Krüger et al. [60], suggesting that highintensity interval training (HIT) sessions are mainly followed by a higher rate of apoptotic highly differentiated T-cells (CD3+/CD28-/CD57+), which are known to affect immunity to control latent infections. In contrast, continuous exercise seems to induce an increase in apoptosis in low differentiated T-cells (CD3+/CD28+/CD57-) and regulatory T-cells (Tregs), which may affect defense against new infectious agents. However, progenitor cell mobilization did not differ between the two exercise groups [60].

After discussing the studies which comprise long-term exercise, the focus is set on acute endurance exercise. The reduction in $\gamma \delta$ T-cells after a cycling exercise in elderly subjects, which was not confounded by the serologic status of CMV, HSV-1, EBV, and PVB19, suggests an age-related impairment of $\gamma \delta$ T-cells mediating immunosurveillance as a response to acute PA [49]. Therefore, it seems unlikely that age-induced $\gamma \delta$ T-cell decline is stimulated by these named latent viral infections [49]. It was already shown that these potent cytotoxic $\gamma \delta$ T-cells are highly responsive to exercise and psychologically induced stress, as well as to $\beta$-agonist infusion [61]. The results suggest that $\gamma \delta$ T-cell mobilization is a response to stressful situations, where antigen exposure is more likely [49]. Generally, acute stress and physical exercise have a notable effect on the reallocation of blood lymphocyte subsets, which is known to be largely mediated by $\beta$-adrenergic mechanisms, especially by $\beta 2$-adrenoceptor positive cells from the marginal pool, and by a demargination of leukocyte reservoirs preserved within blood vessels, the lungs, spleen, and liver [16,62]. Epinephrine probably induces through CD11a and CX3CR1 an adrenergic leucocytosis due to a demargination and a release of these leukocyte subsets into the circulating blood. Because of the cytotoxic effector function of leukocytes expressing this phenotype, immediate protection from pathogens might be provided [62].

In heart failure, $\beta$-adrenergic receptor ( $\beta$-AR) dysfunction plays an important role as well. It has been demonstrated that these molecular alterations and the associated dysregulation of $\beta 1-A R$ and $\beta 2-A R$ can be reversed through exercise [63]. Similar dysfunctions of the $\beta$-AR system have been described in the senescent heart. The blood pressure-lowering effect of aerobic endurance exercise in hypertensive subjects, which is provoked by a reduction in $\beta$-AR dysfunction due to a diminution of sympathetic neural activity, has been reported as one of the main mechanisms explaining the favorable effects of PA [63]. Furthermore, it is suggested that a reduced sensitivity of $\beta 2$-ARs and a decreased thymic output during aging are attributed to fewer mobilized naïve CD8+ T-cells in elderly subjects, which negatively affects catecholamine-induced cell restructuring [64].

This effect might be influenceable with PA, as Brown et al. [52] showed for trained subjects higher proportions of naïve CD8+ cells after a single bout of treadmill exercise. Additionally, senescent T-cell subsets were decreased, supporting the theory that exercise frees up immune space for naïve T-cells [65]. Secondary, the authors highlighted important sex and training status differences in senescent and naïve T-cells. A potential explanation for sex differences could be body composition (e.g., the in average higher body fat percentage in females) or hormone appearance, namely estrogen concentrations. The authors also suggested a sex-specific effect of training status on senescent T-cells, but the sex differences could not be fully explained by the data set [52].

Philippe et al.'s [50] data supports the theory of PA-induced free immune space and shift to lower differentiated cell types by upregulation of naïve and CM CD8+ T-cells 
through concentric and eccentric walking exercise. The increased CD4+/CD8+ ratio, mainly caused by higher levels of CD4+ T-cells, indicates that the commonly reduced CD4+/CD8+ ratio in the aging process could be affected by exercise training [50]. This rise in CD4+ T-cells may be caused by the mobilization of hematopoietic stem cells into peripheral blood due to acute or chronic exercise. Hematopoietic stem and progenitor cells (HSPC) are responsible for sustaining all mature blood cells, and their activity is directed by the bone marrow niche. It is suggested that an acute bout of exercise is a potent physiological stressor inducing the mobilization and proliferation of HSPC populations [66]. It is validated that, e.g., increasing levels of Granulocyte colony-stimulating factor (G-CSF), IL-6, and Monocyte Chemoattractant Protein-1 (MCP-1) triggers the mobilization of HSPCs into the blood plasma [67]. Furthermore, the $\alpha$-chemokine stromal-derived factor 1 (SDF-1), bioactive Phosphosphingolipids (S1P and C1P), and Adenosine triphosphate (ATP) seam to play a major role in the migration of HSPCs [68].

Those changes may take $24-48 \mathrm{~h}$ to be detected in HSPC proportions. There seems to be a relationship between HSPCs and exercise-induced norepinephrine increase. Possibly, during or after acute exercise, more differentiated HSPCs migrate to peripheral tissues, e.g., to muscle tissue, to promote tissue repair [66]. HSPCs may migrate to the thymus for differentiation to naïve CD4+ cells, or more differentiated T-cells are mobilized from the marginal pool and the spleen, which might both contribute to modified CD4+ cell proportion $[64,69]$. Interestingly, there were no remarkable differences found between the concentric and eccentric exercise groups for T-cell subsets. Thus, uphill or downhill walking may be equally regarding positive alterations of immunosenescence, and therefore both exercise regimens can be recommended for elderly persons, especially for pre-diabetic subjects. Additionally, CMV serostatus was analyzed in this study but could be excluded as a confounder [50]. Results revealed that exercise had a positive effect on immune health irrespective of viral serostatus in this survey.

The impact of PA on cellular senescence and the immune system, in general, is best investigated for endurance exercise. Nevertheless, some data exist about immunological changes with resistance exercise training. In this review, three studies were included examining resistance exercise interventions exclusively. Neves et al. [47] concluded that acute effects of resistance exercise conducted at $50 \%$ and $80 \%$ of $1 \mathrm{RM}$, compared to no exercise, did not have suppressive effects on immune parameters. Though, the authors found an increase in IgA levels after resistance exercise sessions, which suggests more efficient protection against respiratory infections in elderly women [47]. Dinh et al. [33] found similar results for strength endurance training ( 2 sets of 30 repetitions at $40 \%$ of $1 \mathrm{RM})$ compared with intensive strength training as for moderate vs. intense aerobic exercise training. Strength endurance training significantly reduced senescence-prone T-cells, whereas a control group and the intensive strength training groups showed no differences. This could also be explained by the exercise intensity-dependent mobilization of T-cells into the bloodstream, which is induced by the lymphocyte mobilization through $\beta 2$-AR by hormones released during exercise as described previously [64]. It can be assumed that high-intensity resistance exercise causes a greater mobilization of senescenceprone T-cells into the blood than moderate-intensity exercise [11]. Therefore, training protocols comprising many repetitions with sufficient external resistance may be more appropriate to reduce senescent cells in elderly women [33]. Conversely, Raso et al. [53] found no significant differences in any phenotypic or functional immune parameters after a 12-month moderate resistance exercise intervention in elderly women but an increase in muscle strength. Similar outcomes were published by Flynn et al. [70], who as well did not find an immune suppression after a single bout of resistance exercise or a change in immunological parameters after 10 weeks of resistance training in elderly women. Overall, the current state of research is controversial regarding the effects of resistance exercise on immune function. This may be related to the selection of participants, training strategies, and ethnic or geographical differences [53]. Furthermore, it can be hypothesized that there could be differences in total workload conducted from individuals performing 
resistance training [47]. Dohi et al. [71] demonstrated that female subjects performing the same relative exercise intensity might have distinct immune responses due to differences in absolute total work performance. It is conspicuous that apparently, mainly female individuals were examined for resistance exercise surveys, which could have an impact on immune system parameters, due to described hormonal and body composition differences. In the studies included in this systematic analysis, only elderly, supposable postmenopausal women were investigated. Therefore, the suggested effects of the menstrual cycle on response to resistance exercise [72] likely did not play any role in named studies. However, it is known that loss of sex hormones due to aging results in an unclear mechanism of reduced immune function. The supposed sex-specific failing of the immune system needs to be determined in further studies [73].

Indirect comparison of aerobic versus resistance exercise, the considered study of Abd El-Kader et al. [48] also provides evidence that aerobic exercise seems to be more appropriate for modulation of the immune system and inflammatory markers in elderly individuals. In both exercise groups, lymphocyte numbers increased, and the CD4+/CD8+ ratio decreased, but only in the aerobic group, these changes were significant after 6 months of regular training [48]. There are also data on other forms of physical activity and immune aging, such as the increased activity of daily living or a lifelong active lifestyle [74,75]. However, these were not included in the review due to a lack of comparability.

For the additional outcome measure 'telomere length', Silva et al. [51] found greater telomere lengths in trained groups. In blood leukocytes of young and middle-aged endurance athletes, the authors found an increase in telomerase activity and expression of telomere stabilizing proteins, as well as downregulation of cell-cycle inhibitors compared to untrained subjects. Long-term continuous exercising attenuated telomere erosion in the leukocytes of examined middle-aged athletes. In conclusion, PA is suggested to be a powerful intervention to upregulate telomere-stabilizing proteins in mice and in humans and protects from stress-induced vascular apoptosis [76]. In contrast to these results, Puterman et al. [77] could not find a direct effect of PA on telomere length in elderly female caregivers experiencing chronic stress, but they did show that PA buffers telomere erosion caused by stress in investigated subjects [77]. Recent studies, such as Najarro et al. [78], approached the issue of the importance of telomere length for the quality of the immune response. They found a positive association of robust antibody response to telomere length of lymphocytes and no association between telomere length and monocyte antigen-presenting cell function. The results suggest that the adaptive immune response is more affected by age-associated effects, and consequently, is an adequate indicator for the state of immunosenescence [78]. The findings regarding a more important role of adaptive immunity in cellular senescence coincide with the results of previous investigations [16,17]. In a direct comparison of aerobic endurance training, HIT, and resistance training, it has been shown that each training protocol seems to induce specific cellular pathways in circulating leukocytes and likely leads to a differential impact on cellular aging [79]. Endurance training and HIT may increase telomerase activity and telomere length, which is important for cellular senescence, regenerative capacity, and, therefore, healthy aging. Resistance exercise showed no effect on these parameters. These findings identify telomerase activity and telomere length as potential and sensitive cellular parameters to quantify the preventive effects of exercise interventions. Generally, individual training recommendations guided by measurement of these and other potential parameters associated with cellular senescence and training response may lead to improvement of adherence and efficacy of PA and training programs in cardiovascular disease prevention [79].

Finally, some limitations of this review need to be pointed out. Overall, only two databases were screened, so studies published elsewhere may have been missed. Only studies that were accessible via the institution and open access were included to guarantee the reproducibility of the search, even if this could have excluded relevant results. Furthermore, the small sample size in most of the studies limits the generalizability and evidence of described outcomes. Further limitations are revealed by the tools applied for 
assessing the risk of bias. Only three studies were RCTs, which limits the generalizability of the results. The RoB2 Tool [44] applied for the analyzed RCTs showed that mostly the assignment to the groups was not clearly described, as well as missing data. Therefore, only the study of Raso et al. [53] had a low risk of bias. Regarding the non-RCTs, the appropriate ROBINS-I Tool [45] was used to analyze them. It must be stated that all studies showed some concerns. Pistillo et al. [49] even showed a serious risk due to unclear risk of bias for some domains. This is probably caused by study design because Pistillo et al. [49] investigated retrospectively. The concerns regarding studies analyzed by the ROBINS-I tool are mainly caused by the study designs, which are not randomized or controlled and therefore have a lower evidence grade and validity. Moreover, most studies failed to blind subjects or investigators.

Besides, not all studies were supervised, so it is unclear if the participants conducted their exercise program as instructed by the authors. Some of the studies did not monitor diet or additional PA during the study phase. Even though it is known that diet and regular PA may have an impact on immunological parameters and the effectiveness of the immune system [80]. Additionally, the two included retrospective studies are based on self-reporting and questionnaires and therefore are limited in their validity. As already mentioned, in the resistance exercise studies, only women were investigated, which can be a potential bias. It is noticeable that mainly adaptive immune responses and especially T-cell subsets were analyzed in all the described studies. This supports the theory that the effects of PA on cells belonging to the adaptive immune response were analyzed more frequently.

Regardless, there is a need for more studies investigating innate immunity and its cellular subsets. The dimensions of exercise-induced immune changes could be different between studies due to different training protocols, methodologies, ages, sex, and the testing procedures themselves [8].

In terms of the testing procedures, the heterogenic staining methods and investigated clusters of differentiation to detect similar cells also can be identified as a general confounding factor. Various approaches to identify possibly important subpopulations in included studies, and in general, in immune system studies, were used to measure the same cells. For example, NK cells were analyzed only via CD16+ [50] or additionally subdivided into CD56 $6^{\mathrm{dim}}$ and CD56 ${ }^{\text {bright }}$ NK cell subsets [53]. Senescent and differentiated cells were measured differently in examined studies as well. Dinh et al. [33] distinguished naïve, memory, and senescent T-cells via CD28 and CD57. However, Philippe et al. [50] differed between naïve, CM, and TEMRA T-cells via CCR7 and CD45RO. Consequently, it is unclear to what extent the methodological differences affect the results of this work. So far, there are no standardized procedures regarding the collection of leukocyte subpopulations. Therefore, there is a need to standardize methodologies and nomenclatures, which would enable merging results from studies conducted by different research groups and comparing various populations. The inconsistency reporting results in absolute or relative numbers, such as total counts of leukocyte subsets or percentage, may contribute to existent inconsistencies in literature [81]. A standardized way of expressing data might be conductive to gain manifest data and a more definite view on relationships and processes of cellular senescence and its interaction with PA.

Whether or not temporary changes in the percentage of leukocyte subpopulations, especially in T-cells, can be a prognostic biomarker is not completely established yet [31]. The number of leukocytes and their subtypes in the peripheral blood is influenced by cell traffic to and from different tissues. Therefore, the composition of, for example, T-cells in peripheral blood may not be representative of subsets and functionality of T-cells present in other tissues in the body [31]. Furthermore, circulating leukocytes are a major source of cytokines and can, for example, infiltrate adipose tissue in response to obesity. Therefore, it may be a possibility that leukocytes function in addition as biomarkers for identifying morbid forms of obesity [81]. In conclusion, it seems like leukocyte subsets percentage in peripheral blood may not always be a valid measuring object. 
Altogether, there is evidence-based research results on PA and cellular senescence of leukocyte subpopulations. PA has the potential to improve immune function as well as health outcomes and therefore is a behavioral intervention that could be applied in prevention and therapy in subjects of all ages [7]. Thereby, exercise may delay or prevent the transition from the non-IRP into the IRP group. Furthermore, exercise is apparently able to revert a sedentary individual from the IRP to the non-IRP status, called immune restoration [27]. However, future research is required to support the theory of PA for the prevention of cellular senescence or restoration of an intact immune system. There is a need for RCT studies and studies with more adequately control groups. Additionally, prospective longitudinal studies could help to examine the long-term effects of PA over the lifespan. Though, it is ethically questionable to design a long-term RCT with a sedentary control group.

An innovative, fast-growing research field describes these degenerative or adaptive changes in the immune system due to extrinsic or intrinsic stressors during the aging process from a genetic perspective to gain a better understanding of the complexity of the inter-relatedness of immunosenescence, inflammation, and aging [82]. This means the current research regarding immune aging is more and more integrating omics-based research and system biology approaches. Age-related changes were increasingly examined via genomics, metagenomics, transcriptomics, and metabolomics using molecular profiling methods. These technologies have been used to analyze the role of T-cells in aging and used outcomes such as mitochondrial DNA heteroplasmy, DNA methylation, miRNA expression, serum metabolite levels, and metagenomic diversity, as well as the composition of the gut microbiome. These molecular factors were applied to aging-focused research in combination with the environment, like diet or lifestyle, in various study designs [82].

A more global sight on exercise and PA reveals that physical inactivity is becoming a major public health problem worldwide [55]. In the face of the current and possible future global spread of different kinds of infectious diseases, like the coronavirus disease 2019 (COVID-19), it is even more important to promote an active lifestyle in young and elderly individuals to probably prevent infectious and chronic diseases associated with aging and cellular senescence [83]. PA cannot entirely reverse aging processes, but it can attenuate damaging cellular and systemic mechanisms and, therefore, improve health substantially [55].

\section{Conclusions}

Overall, the systematic analysis of current evidence showed mixed results regarding cellular senescence and the impact of PA on different leukocyte subpopulations. Though, the studies showed that PA does have an influence on cellular senescence. Results revealed that the type of exercise, as well as the duration and persistence of exercising differentially modulate immunosenescence. Exercise interventions in studies were heterogenic. Hence, there is no explicit evidence to enunciate recommendations for PA, which particularly delays, prevents, or slows down cellular senescence. However, high-intensity endurance exercise may affect immunosenescence in a negative way by leading to the accumulation of senescent cells. Therefore, it is suggested that moderate-intensity endurance exercise is more suitable to influence cellular senescence in a positive way by mobilizing higher proportions of T-helper cells, inducing beneficial modifications in proportions of naïve and memory T-cell subsets, as well as decreasing proportions of senescent cells. In particular, the TEMRA subsets decrease for both T-helper and cytotoxic T-cells. On the contrary, resistance exercise is suggested to be less effective and not as extensively modulating the immune system as aerobic endurance exercise. In the strength-focused studies, no significant effects on immune parameters could be detected. Yet, moderate strength endurance training seems to be more suitable than intensive strength training to promote a shift in immune parameters. Further human-based RCTs and longitudinal research is required to clarify presented results. The impact of sex on the immune system still needs to be investigated, 
and there is a necessity to develop standardized measurement methods for immune cells, as well as homogeneous exercise interventions in relation to cellular senescence.

Author Contributions: Conceptualization, L.B. and K.A.; methodology, L.B. and K.A.; validation, K.K., C.W. and K.A.; formal analysis, L.B., K.K., C.W. and K.A.; investigation, L.B., K.K., C.W. and K.A.; resources, K.K.; data curation, K.K., C.W. and K.A.; writing-original draft preparation, L.B.; writing-review and editing, K.K., C.W. and K.A. All authors have read and agreed to the published version of the manuscript.

Funding: This research received no external funding.

Conflicts of Interest: The authors declare no conflict of interest.

\section{Appendix A}

Table A1. Database research of included studies.

\begin{tabular}{|c|c|c|c|}
\hline \multicolumn{4}{|c|}{ Date: 26.07.2020; Database: Pubmed } \\
\hline No. & Title & Authors & Year \\
\hline 1 & $\begin{array}{l}\text { Inflammatory cytokines and immune } \\
\text { system modulation by aerobic versus } \\
\text { resisted exercise training for elderly }\end{array}$ & $\begin{array}{c}\text { Abd El-Kader, Shehab M.\& Al-Shreef, } \\
\text { Fadwa M. }\end{array}$ & 2018 \\
\hline 2 & $\begin{array}{c}\text { Strength Endurance Training but Not } \\
\text { Intensive Strength Training Reduces } \\
\text { Senescence-Prone T Cells in } \\
\text { Peripheral Blood in } \\
\text { Community-Dwelling Elderly } \\
\text { Women }\end{array}$ & $\begin{array}{c}\text { Cao Dinh, Hung; Njemini, Rose; } \\
\text { Onyema, Oscar Okwudiri; Beyer, Ingo; } \\
\text { Liberman, Keliane; Dobbeleer, Liza de; } \\
\text { Renmans, Wim; Vander Meeren, Sam; } \\
\text { Jochmans, Kristin; Delaere, Andreas; } \\
\text { Knoop, Veerle; Bautmans, Ivan }\end{array}$ & 2019 \\
\hline 3 & $\begin{array}{l}\text { Resistance exercise sessions do not } \\
\text { provoke acute immunosuppression } \\
\text { in older women }\end{array}$ & $\begin{array}{l}\text { Neves, Sergio da Cunha Jr; Lima, Ricardo } \\
\text { Moreno; Simões, Herbert Gustavo; } \\
\text { Marques, Mario C.; Reis, Victor Machado; } \\
\text { Oliveira, Ricardo Jacó de }\end{array}$ & 2009 \\
\hline \multicolumn{4}{|c|}{ Date: 27-29.07.2020; Database: Web of Science } \\
\hline 4 & $\begin{array}{l}\text { Concentric and Eccentric Endurance } \\
\text { Exercise Reverse Hallmarks of T-Cell } \\
\text { Senescence in Pre-diabetic Subjects }\end{array}$ & $\begin{array}{l}\text { Philippe, Marc; Gatterer, Hannes; } \\
\text { Burtscher, Martin; Weinberger, Birgit; } \\
\text { Keller, Michael; Grubeck-Loebenstein, } \\
\text { Beatrix; Fleckenstein, Johannes; Alack, } \\
\text { Katharina; Krüger, Karsten }\end{array}$ & 2019 \\
\hline 5 & $\begin{array}{l}\text { Moderate and intense exercise } \\
\text { lifestyles attenuate the effects of } \\
\text { aging on telomere length and the } \\
\text { survival and composition of T cell } \\
\text { subpopulations }\end{array}$ & $\begin{array}{l}\text { Rodrigues Silva, Leia Cristina; Araujo, } \\
\text { Adriana Ladeira de; Fernandes, Juliana } \\
\text { Ruiz; Toledo Matias, Manuella de Sousa; } \\
\text { Silva, Paulo Roberto; Duarte, Alberto J. S.; } \\
\text { Garcez Leme, Luiz Eugenio; Benard, Gil }\end{array}$ & 2016 \\
\hline 6 & $\begin{array}{l}\text { The effects of age and viral serology } \\
\text { on gamma delta T-cell numbers and } \\
\text { exercise responsiveness in humans }\end{array}$ & $\begin{array}{l}\text { Pistillo, Mira; Bigley, Austin B.; } \\
\text { Spielmann, Guillaume; LaVoy, Emily C.; } \\
\text { Morrison, Mark R.; Kunz, Hawley; } \\
\text { Simpson, Richard J. }\end{array}$ & 2013 \\
\hline 7 & $\begin{array}{l}\text { Training status and sex influence on } \\
\text { senescent T-lymphocyte } \\
\text { redistribution in response to acute } \\
\text { maximal exercise }\end{array}$ & $\begin{array}{l}\text { Brown, Frankie F.; Bigley, Austin B.; } \\
\text { Sherry, Chris; Neal, Craig M.; Witard, } \\
\text { Oliver C.; Simpson, Richard J.; Galloway, } \\
\text { Stuart D. R. }\end{array}$ & 2014 \\
\hline 8 & $\begin{array}{l}\text { Frequent participation in high } \\
\text { volume exercise throughout life is } \\
\text { associated with a more differentiated } \\
\text { adaptive immune response }\end{array}$ & $\begin{array}{l}\text { Moro-García, Marco Antonio; } \\
\text { Fernández-García, Benjamín; Echeverría, } \\
\text { Ainara; Rodríguez-Alonso, Manuel; } \\
\text { Suárez-García, Francisco Manuel; } \\
\text { Solano-Jaurrieta, Juan José; López-Larrea, } \\
\text { Carlos; Alonso-Arias, Rebeca }\end{array}$ & 2014 \\
\hline 9 & $\begin{array}{l}\text { Effect of Resistance Training on } \\
\text { Immunological Parameters of } \\
\text { Healthy Elderly Women }\end{array}$ & $\begin{array}{c}\text { Raso, Vagner; Benard, Gil; DA Silva } \\
\text { Duarte, Alberto José; Natale, Valéria } \\
\text { Maria }\end{array}$ & 2007 \\
\hline
\end{tabular}


Table A2. Summary of selected investigated physical characteristics in each study.

\begin{tabular}{|c|c|c|c|c|c|c|c|c|}
\hline Author & Age & Sex & CMV Serostatus & BMI & $\mathrm{VO}_{2 \max }$ & HR & Training Volume & Lactate \\
\hline [48] Abd El-Kader et al. & $\checkmark$ & $x$ & $\mathrm{x}$ & $\checkmark$ & $\mathrm{x}$ & $\checkmark$ & $\checkmark$ & $x$ \\
\hline [52] Brown et al. & $\checkmark$ & $\checkmark$ & $\checkmark$ & $x$ & $x$ & $\checkmark$ & $\checkmark$ & $\checkmark$ \\
\hline [33] Dinh et al. & $\checkmark$ & $\checkmark$ & $\checkmark$ & $\checkmark$ & $x$ & $\mathrm{x}$ & $\checkmark$ & $x$ \\
\hline [54] Moro-García et al. & $\checkmark$ & $\checkmark$ & $\checkmark$ & $\checkmark$ & $\checkmark$ & $\mathrm{x}$ & $\checkmark$ & $x$ \\
\hline [47] Neves et al. & $\checkmark$ & $\checkmark$ & $\mathrm{x}$ & $\checkmark$ & $\mathrm{x}$ & $\mathrm{x}$ & $\checkmark$ & $\mathrm{x}$ \\
\hline [50] Philippe et al. & $\checkmark$ & $\checkmark$ & $x$ & $\mathrm{x}$ & $\mathrm{x}$ & $\mathrm{x}$ & $\checkmark$ & $\mathrm{x}$ \\
\hline [49] Pistillo et al. & $\checkmark$ & $\checkmark$ & $\checkmark$ & $\checkmark$ & $\checkmark$ & $\checkmark$ & $\checkmark$ & $\mathrm{x}$ \\
\hline [53] Raso et al. & $\checkmark$ & $\checkmark$ & $\checkmark$ & $\checkmark$ & $\mathrm{x}$ & $\mathrm{x}$ & $\checkmark$ & $x$ \\
\hline [51] Silva et al. & $\checkmark$ & $x$ & $x$ & $\checkmark$ & $\mathrm{x}$ & $x$ & $\checkmark$ & $x$ \\
\hline
\end{tabular}

BMI: body mass index; CMV: cytomegalovirus; HR: heart rate; $\mathrm{VO}_{2 \max }$ : maximal oxygen uptake.

\section{References}

1. Bektas, A.; Schurman, S.H.; Sen, R.; Ferrucci, L. Human T cell immunosenescence and inflammation in aging. J. Leukoc. Biol. 2017, 102, 977-988. [CrossRef]

2. Montecino-Rodriguez, E.; Berent-Maoz, B.; Dorshkind, K. Causes, consequences, and reversal of immune system aging. J. Clin. Investig. 2013, 123, 958-965. [CrossRef]

3. de Araújo, A.L.; Silva, L.C.; Fernandes, J.R.; Benard, G. Preventing or reversing immunosenescence: Can exercise be an immunotherapy? Immunotherapy 2013, 5, 879-893. [CrossRef] [PubMed]

4. Garatachea, N.; Pareja-Galeano, H.; Sanchis-Gomar, F.; Santos-Lozano, A.; Fiuza-Luces, C.; Morán, M.; Emanuele, E.; Joyner, M.J.; Lucia, A. Exercise attenuates the major hallmarks of aging. Rejuvenation Res. 2015, 18, 57-89. [CrossRef]

5. Simpson, R.J.; Lowder, T.W.; Spielmann, G.; Bigley, A.B.; LaVoy, E.C.; Kunz, H. Exercise and the aging immune system. Ageing Res. Rev. 2012, 11, 404-420. [CrossRef] [PubMed]

6. Pawelec, G.; Larbi, A.; Derhovanessian, E. Senescence of the human immune system. J. Comp. Pathol. 2010, 142 (Suppl. 1), $39-44$. [CrossRef] [PubMed]

7. Alack, K.; Pilat, C.; Krüger, K. Current knowledge and new challenges in exercise immunology. Dtsch. Z. Sportmed. 2019, 70, 250-260. [CrossRef]

8. Sellami, M.; Gasmi, M.; Denham, J.; Hayes, L.D.; Stratton, D.; Padulo, J.; Bragazzi, N. Effects of Acute and Chronic Exercise on Immunological Parameters in the Elderly Aged: Can Physical Activity Counteract the Effects of Aging? Front. Immunol. 2018, 9, 2187. [CrossRef]

9. Kovaiou, R.D.; Grubeck-Loebenstein, B. Age-associated changes within CD4+ T cells. Immunol. Lett. 2006, 107, 8-14. [CrossRef]

10. Wadhwa, R.; Kaul, Z.; Kaul, S.C. Cell Cycle Checkpoints and Senescence. In Cellular Ageing and Replicative Senescence; Rattan, S., Hayflick, L., Eds.; Springer: Cham, Switzerland, 2016; pp. 145-167.

11. Kirkland, J.L.; Tchkonia, T. Cellular Senescence: A Translational Perspective. EBioMedicine 2017, 21, 21-28. [CrossRef]

12. LeBrasseur, N.K.; Tchkonia, T.; Kirkland, J.L. Cellular Senescence and the Biology of Aging, Disease, and Frailty. Frailty Pathophysiol. Phenotype Patient Care 2015, 83, 11-18. [CrossRef]

13. Rayess, H.; Wang, M.B.; Srivatsan, E.S. Cellular senescence and tumor suppressor gene p16. Int. J. Cancer 2012, 130, 1715-1725. [CrossRef]

14. Vargas, J.; Feltes, B.C.; Poloni Jde, F.; Lenz, G.; Bonatto, D. Senescence; an endogenous anticancer mechanism. Front. Biosci. 2012, 17, 2616-2643. [CrossRef] [PubMed]

15. Fulop, T.; Larbi, A.; Dupuis, G.; Le Page, A.; Frost, E.H.; Cohen, A.A.; Witkowski, J.M.; Franceschi, C. Immunosenescence and Inflamm-Aging As Two Sides of the Same Coin: Friends or Foes? Front. Immunol. 2017, 8, 1960. [CrossRef]

16. Walsh, N.P.; Gleeson, M.; Shephard, R.J.; Gleeson, M.; Woods, J.A.; Bishop, N.C.; Fleshner, M.; Green, C.; Pedersen, B.K.; Hoffman-Goetz, L.; et al. Position statement. Part one: Immune function and exercise. Exerc. Immunol. Rev. 2011, 17, 6-63.

17. Sansoni, P.; Vescovini, R.; Fagnoni, F.; Biasini, C.; Zanni, F.; Zanlari, L.; Telera, A.; Lucchini, G.; Passeri, G.; Monti, D.; et al. The immune system in extreme longevity. Exp. Gerontol. 2008, 43, 61-65. [CrossRef] [PubMed]

18. Pawelec, G.; Derhovanessian, E.; Larbi, A.; Strindhall, J.; Wikby, A. Cytomegalovirus and human immunosenescence. Rev. Med. Virol. 2009, 19, 47-56. [CrossRef] [PubMed]

19. Morrisette-Thomas, V.; Cohen, A.A.; Fülöp, T.; Riesco, É.; Legault, V.; Li, Q.; Milot, E.; Dusseault-Bélanger, F.; Ferrucci, L. Inflamm-aging does not simply reflect increases in pro-inflammatory markers. Mech. Ageing Dev. 2014, 139, 49-57. [CrossRef] [PubMed]

20. Macaulay, R.; Akbar, A.N.; Henson, S.M. The role of the T cell in age-related inflammation. Age 2013, 35, 563-572. [CrossRef] [PubMed] 
21. World Health Organization. Global Recommendations on Physical Activity for Health; World Health Organization: Geneva, Switzerland, 2010; ISBN 9789241599979.

22. Campbell, J.P.; Turner, J.E. Debunking the Myth of Exercise-Induced Immune Suppression: Redefining the Impact of Exercise on Immunological Health across the Lifespan. Front. Immunol. 2018, 9, 648. [CrossRef] [PubMed]

23. Matthews, C.E.; Ockene, I.S.; Freedson, P.S.; Rosal, M.C.; Merriam, P.A.; Hebert, J.R. Moderate to vigorous physical activity and risk of upper-respiratory tract infection. Med. Sci. Sports Exerc. 2002, 34, 1242-1248. [CrossRef] [PubMed]

24. Nieman, D.C. Exercise, infection, and immunity. Int. J. Sports Med. 1994, 15, 131-141. [CrossRef] [PubMed]

25. Nieman, D.C.; Henson, D.A.; Austin, M.D.; Sha, W. Upper respiratory tract infection is reduced in physically fit and active adults. Br. J. Sports Med. 2011, 45, 987-992. [CrossRef]

26. Krüger, K.; Mooren, F.-C.; Pilat, C. The Immunomodulatory Effects of Physical Activity. Curr. Pharm. Des. 2016, 22, 3730-3748. [CrossRef]

27. Simpson, R.J.; Guy, K. Coupling aging immunity with a sedentary lifestyle: Has the damage already been done?-A mini-review. Gerontology 2010, 56, 449-458. [CrossRef] [PubMed]

28. Moher, D.; Liberati, A.; Tetzlaff, J.; Altman, D.G. Preferred Reporting Items for Systematic Reviews and Meta-Analyses: The PRISMA Statement. PLoS Med. 2009, 6, e1000097. [CrossRef]

29. Weyand, C.M.; Goronzy, J.J. Aging of the Immune System. Mechanisms and Therapeutic Targets. Ann. Am. Thorac. Soc. 2016, 13, 422-428. [CrossRef] [PubMed]

30. Chilton, W.L.; Marques, F.Z.; West, J.; Kannourakis, G.; Berzins, S.P.; O’Brien, B.J.; Charchar, F.J. Acute exercise leads to regulation of telomere-associated genes and microRNA expression in immune cells. PLoS ONE 2014, 9, e92088. [CrossRef]

31. Cosgrove, C.; Galloway, S.D.R.; Neal, C.; Hunter, A.M.; McFarlin, B.K.; Spielmann, G.; Simpson, R.J. The impact of 6-month training preparation for an Ironman triathlon on the proportions of naive, memory and senescent T cells in resting blood. Eur. J. Appl. Physiol. 2012, 112, 2989-2998. [CrossRef]

32. Dinh, H.C.; Bautmans, I.; Beyer, I.; Onyema, O.O.; Liberman, K.; De Dobbeleer, L.; Renmans, W.; Vander Meeren, S.; Jochmans, K.; Delaere, A.; et al. Six weeks of strength endurance training decreases circulating senescence-prone T-lymphocytes in cytomegalovirus seropositive but not seronegative older women. Immun. Ageing 2019, 16, 17. [CrossRef]

33. Dinh, H.C.; Njemini, R.; Onyema, O.O.; Beyer, I.; Liberman, K.; De Dobbeleer, L.; Renmans, W.; Vander Meeren, S.; Jochmans, K.; Delaere, A.; et al. Strength Endurance Training but Not Intensive Strength Training Reduces Senescence-Prone T Cells in Peripheral Blood in Community-Dwelling Elderly Women. J. Gerontol. A Biol. Sci. Med. Sci. 2019, 74, 1870-1878. [CrossRef]

34. Kapasi, Z.F.; Ouslander, J.G.; Schnelle, J.F.; Kutner, M.; Fahey, J.L. Effects of an exercise intervention on immunologic parameters in frail elderly nursing home residents. J. Gerontol. A Biol. Sci. Med. Sci. 2003, 58, 636-643. [CrossRef] [PubMed]

35. Karim, A.A.; Jabbar, B. Effects of hypobaric Endurance Training on Graded Exercise Induced Lymphocyte Mobilization, Senescence and Their Surface Thiol Levels in Elite Male Athletes. Int. J. Appl. Exerc. Physiol. 2018, 7, 48-55. [CrossRef]

36. Kohut, M.L.; Senchina, D.S.; Madden, K.S.; Martin, A.E.; Felten, D.L.; Moynihan, J.A. Age effects on macrophage function vary by tissue site, nature of stimulant, and exercise behavior. Exp. Gerontol. 2004, 39, 1347-1360. [CrossRef] [PubMed]

37. Mota, M.P.; Peixoto, F.M.; Soares, J.F.; Figueiredo, P.A.; Leitao, J.C.; Gaivao, I.; Duarte, J.A. Influence of aerobic fitness on age-related lymphocyte DNA damage in humans: Relationship with mitochondria respiratory chain and hydrogen peroxide production. AGE 2010, 32, 337-346. [CrossRef] [PubMed]

38. Ai-jun, N.; Yan-qun, W.; Jin-long, L. Effect of healthy Qigong "WuQinXi" exercise on peripheral blood T-cell subgroups in middle-aged subjects. Afr. J. Biotechnol. 2010, 9, 4620-4623.

39. Reidy, P.T.; Lindsay, C.C.; McKenzie, A.I.; Fry, C.S.; Supiano, M.A.; Marcus, R.L.; LaStayo, P.C.; Drummond, M.J. Aging-related effects of bed rest followed by eccentric exercise rehabilitation on skeletal muscle macrophages and insulin sensitivity. Exp. Gerontol. 2018, 107, 37-49. [CrossRef]

40. Soares, J.P.; Mota, M.P.; Duarte, J.A.; Collins, A.; Gaivao, I. Age-related increases in human lymphocyte DNA damage: Is there a role of aerobic fitness? Cell Biochem. Funct. 2013, 31, 743-748. [CrossRef] [PubMed]

41. Spielmann, G.; McFarlin, B.K.; O'Connor, D.P.; Smith, P.J.W.; Pircher, H.; Simpson, R.J. Aerobic fitness is associated with lower proportions of senescent blood T-cells in man. Brain Behav. Immun. 2011, 25, 1521-1529. [CrossRef]

42. Wang, H.Y.; Bashore, T.R.; Tran, Z.V.; Friedman, E. Age-related decreases in lymphocyte protein kinase C activity and translocation are reduced by aerobic fitness. J. Gerontol. A Biol. Sci. Med. Sci. 2000, 55, 545-551. [CrossRef]

43. Wang, J.-S.; Chen, W.-L.; Weng, T.-P. Hypoxic exercise training reduces senescent T-lymphocyte subsets in blood. Brain Behav. Immun. 2011, 25, 270-278. [CrossRef]

44. Higgins, J.P.T.; Altman, D.G.; Gotzsche, P.C.; Juni, P.; Moher, D.; Oxman, A.D.; Savovic, J.; Schulz, K.F.; Weeks, L.; Sterne, J.A.C. The Cochrane Collaboration's tool for assessing risk of bias in randomised trials. BMJ 2011, 343, d5928. [CrossRef]

45. Sterne, J.A.C.; Hernán, M.A.; Reeves, B.C.; Savović, J.; Berkman, N.D.; Viswanathan, M.; Henry, D.; Altman, D.G.; Ansari, M.T.; Boutron, I.; et al. ROBINS-I: A tool for assessing risk of bias in non-randomised studies of interventions. BMJ 2016, i4919. [CrossRef]

46. Palmowski, J.; Reichel, T.; Boßlau, T.K.; Krüger, K. The effect of acute running and cycling exercise on T cell apoptosis in humans: A systematic review. Scand. J. Immunol 2019, e12834. [CrossRef]

47. da Cunha Neves, S., Jr.; Lima, R.M.; Simões, H.G.; Marques, M.C.; Reis, V.M.; de Oliveira, R.J. Resistance exercise sessions do not provoke acute immunosuppression in older women. J. Strength Cond. Res. 2009, 23, 259-265. [CrossRef] [PubMed] 
48. Abd El-Kader, S.M.; Al-Shreef, F.M. Inflammatory cytokines and immune system modulation by aerobic versus resisted exercise training for elderly. Afr. Health Sci. 2018, 18, 120-131. [CrossRef] [PubMed]

49. Pistillo, M.; Bigley, A.B.; Spielmann, G.; LaVoy, E.C.; Morrison, M.R.; Kunz, H.; Simpson, R.J. The effects of age and viral serology on gamma delta T-cell numbers and exercise responsiveness in humans. Cell. Immunol. 2013, 284, 91-97. [CrossRef]

50. Philippe, M.; Gatterer, H.; Burtscher, M.; Weinberger, B.; Keller, M.; Grubeck-Loebenstein, B.; Fleckenstein, J.; Alack, K.; Krueger, K. Concentric and Eccentric Endurance Exercise Reverse Hallmarks of T-Cell Senescence in Pre-diabetic Subjects. Front. Physiol. 2019, 10. [CrossRef] [PubMed]

51. Silva, L.C.R.; de Araujo, A.L.; Fernandes, J.R.; Matias, M.D.; Silva, P.R.; Duarte, A.J.S.; Garcez Leme, L.E.; Benard, G. Moderate and intense exercise lifestyles attenuate the effects of aging on telomere length and the survival and composition of $\mathrm{T}$ cell subpopulations. AGE 2016, 38, 24. [CrossRef] [PubMed]

52. Brown, F.F.; Bigley, A.B.; Sherry, C.; Neal, C.M.; Witard, O.C.; Simpson, R.J.; Galloway, S.D.R. Training status and sex influence on senescent T-lymphocyte redistribution in response to acute maximal exercise. Brain Behav. Immun. 2014, 39, 152-159. [CrossRef]

53. Raso, V.; Benard, G.; DA Silva Duarte, A.J.; Natale, V.M. Effect of Resistance Training on Immunological Parameters of Healthy Elderly Women. Med. Sci. Sports Exerc. 2007, 39, 2152-2159. [CrossRef] [PubMed]

54. Moro-García, M.A.; Fernández-García, B.; Echeverría, A.; Rodríguez-Alonso, M.; Suárez-García, F.M.; Solano-Jaurrieta, J.J.; López-Larrea, C.; Alonso-Arias, R. Frequent participation in high volume exercise throughout life is associated with a more differentiated adaptive immune response. Brain Behav. Immun. 2014, 39, 61-74. [CrossRef] [PubMed]

55. Lee, I.-M.; Shiroma, E.J.; Lobelo, F.; Puska, P.; Blair, S.N.; Katzmarzyk, P.T. Effect of physical inactivity on major non-communicable diseases worldwide: An analysis of burden of disease and life expectancy. Lancet 2012, 380, 219-229. [CrossRef]

56. Pawelec, G.; Koch, S.; Franceschi, C.; Wikby, A. Human immunosenescence: Does it have an infectious component? Ann. N. Y. Acad. Sci. 2006, 1067, 56-65. [CrossRef]

57. Shearer, G.M. Th1/Th2 changes in aging. Mech. Ageing Dev. 1997, 94, 1-5. [CrossRef]

58. Saygin, O.; Karacabey, K.; Ozmerdivenli, R.; Zorba, E.; Ilhan, F.; Bulut, V. Effect of chronic exercise on immunoglobin, complement and leukocyte types in volleyball players and athletes. Neuro Endocrinol. Lett. 2006, 27, 271-276. [PubMed]

59. McFarlin, B.K.; Flynn, M.G.; Phillips, M.D.; Stewart, L.K.; Timmerman, K.L. Chronic resistance exercise training improves natural killer cell activity in older women. J. Gerontol. A Biol. Sci. Med. Sci. 2005, 60, 1315-1318. [CrossRef]

60. Krüger, K.; Alack, K.; Ringseis, R.; Mink, L.; Pfeifer, E.; Schinle, M.; Gindler, K.; Kimmelmann, L.; Walscheid, R.; Muders, K.; et al. Apoptosis of T-Cell Subsets after Acute High-Intensity Interval Exercise. Med. Sci. Sports Exerc. 2016, 48, 2021-2029. [CrossRef] [PubMed]

61. Anane, L.H.; Edwards, K.M.; Burns, V.E.; Zanten, J.J.; Drayson, M.T.; Bosch, J.A. Phenotypic characterization of gammadelta T cells mobilized in response to acute psychological stress. Brain Behav. Immun. 2010, 24, 608-614. [CrossRef]

62. Dimitrov, S.; Lange, T.; Born, J. Selective mobilization of cytotoxic leukocytes by epinephrine. J. Immunol. 2010, $184,503-511$. [CrossRef] [PubMed]

63. Leosco, D.; Parisi, V.; Femminella, G.D.; Formisano, R.; Petraglia, L.; Allocca, E.; Bonaduce, D. Effects of exercise training on cardiovascular adrenergic system. Front. Physiol. 2013, 4, 348. [CrossRef]

64. Krüger, K.; Lechtermann, A.; Fobker, M.; Völker, K.; Mooren, F.C. Exercise-induced redistribution of T lymphocytes is regulated by adrenergic mechanisms. Brain Behav. Immun. 2008, 22, 324-338. [CrossRef] [PubMed]

65. Simpson, R.J. Aging, persistent viral infections, and immunosenescence: Can exercise "make space"? Exerc. Sport Sci. Rev. 2011, 39, 23-33. [CrossRef] [PubMed]

66. Emmons, R.; Niemiro, G.M.; De, L.M. Hematopoiesis with Obesity and Exercise: Role of the Bone Marrow Niche. Exerc. Immunol. Rev. 2017, 23, 82-95. [PubMed]

67. Szade, A.; Szade, K.; Nowak, W.N.; Bukowska-Strakova, K.; Muchova, L.; Gońka, M.; Żukowska, M.; Cieśla, M.; KachamakovaTrojanowska, N.; Rams-Baron, M.; et al. Cobalt protoporphyrin IX increases endogenous G-CSF and mobilizes HSC and granulocytes to the blood. EMBO Mol. Med. 2019, 11, e09571. [CrossRef]

68. Bujko, K.; Cymer, M.; Adamiak, M.; Ratajczak, M.Z. An Overview of Novel Unconventional Mechanisms of Hematopoietic Development and Regulators of Hematopoiesis-A Roadmap for Future Investigations. Stem Cell Rev. Rep. 2019, 15, 785-794. [CrossRef]

69. Mooren, F.C.; Krüger, K. Apoptotic lymphocytes induce progenitor cell mobilization after exercise. J. Appl. Physiol. 2015, 119, 135-139. [CrossRef] [PubMed]

70. Flynn, M.G.; Fahlman, M.; Braun, W.A.; Lambert, C.P.; Bouillon, L.E.; Brolinson, P.G.; Armstrong, C.W. Effects of resistance training on selected indexes of immune function in elderly women. J. Appl. Physiol. 1999, 86, 1905-1913. [CrossRef] [PubMed]

71. Dohi, K.; Mastro, A.M.; Miles, M.P.; Bush, J.A.; Grove, D.S.; Leach, S.K.; Volek, J.S.; Nindl, B.C.; Marx, J.O.; Gotshalk, L.A.; et al. Lymphocyte proliferation in response to acute heavy resistance exercise in women: Influence of muscle strength and total work. Eur. J. Appl. Physiol. 2001, 85, 367-373. [CrossRef]

72. Thompson, B.; Almarjawi, A.; Sculley, D.; de Jonge, X.J. The Effect of the Menstrual Cycle and Oral Contraceptives on Acute Responses and Chronic Adaptations to Resistance Training: A Systematic Review of the Literature. Sports Med. 2020, 50, 171-185. [CrossRef]

73. Ghosh, M.; Rodriguez-Garcia, M.; Wira, C.R. The Immune System in Menopause: Pros and Cons of Hormone Therapy. J. Steroid Biochem. Mol. Biol. 2014, 142, 171-175. [CrossRef] 
74. Wong, G.C.L.; Narang, V.; Lu, Y.; Camous, X.; Nyunt, M.S.Z.; Carre, C.; Tan, C.; Xian, C.H.; Chong, J.; Chua, M.; et al. Hallmarks of improved immunological responses in the vaccination of more physically active elderly females. Exerc. Immunol. Rev. 2019, 25, 20-33. [PubMed]

75. Minuzzi, L.G.; Rama, L.; Chupel, M.U.; Rosado, F.; Dos Santos, J.V.; Simpson, R.; Martinho, A.; Paiva, A.; Teixeira, A.M. Effects of lifelong training on senescence and mobilization of $\mathrm{T}$ lymphocytes in response to acute exercise. Exerc. Immunol. Rev. 2018, 24, 72-84.

76. Werner, C.; Fürster, T.; Widmann, T.; Pöss, J.; Roggia, C.; Hanhoun, M.; Scharhag, J.; Büchner, N.; Meyer, T.; Kindermann, W.; et al. Physical exercise prevents cellular senescence in circulating leukocytes and in the vessel wall. Circulation 2009, 120, $2438-2447$. [CrossRef]

77. Puterman, E.; Lin, J.; Blackburn, E.; O’Donovan, A.; Adler, N.; Epel, E. The power of exercise: Buffering the effect of chronic stress on telomere length. PLoS ONE 2010, 5, e10837. [CrossRef] [PubMed]

78. Najarro, K.; Nguyen, H.; Chen, G.; Xu, M.; Alcorta, S.; Yao, X.; Zukley, L.; Metter, E.J.; Truong, T.; Lin, Y.; et al. Telomere Length as an Indicator of the Robustness of B- and T-Cell Response to Influenza in Older Adults. J. Infect. Dis. 2015, 212, 1261-1269. [CrossRef] [PubMed]

79. Werner, C.M.; Hecksteden, A.; Morsch, A.; Zundler, J.; Wegmann, M.; Kratzsch, J.; Thiery, J.; Hohl, M.; Bittenbring, J.T.; Neumann, F.; et al. Differential effects of endurance, interval, and resistance training on telomerase activity and telomere length in a randomized, controlled study. Eur. Heart J. 2019, 40, 34-46. [CrossRef]

80. Clements, S.J.; Carding, S.R. Diet, the intestinal microbiota, and immune health in aging. Crit. Rev. Food Sci. Nutr. 2018, 58, 651-661. [CrossRef] [PubMed]

81. Pecht, T.; Gutman-Tirosh, A.; Bashan, N.; Rudich, A. Peripheral blood leucocyte subclasses as potential biomarkers of adipose tissue inflammation and obesity subphenotypes in humans. Obes. Rev. 2014, 15, 322-337. [CrossRef]

82. Valdes, A.M.; Glass, D.; Spector, T.D. Omics technologies and the study of human ageing. Nat. Rev. Genet. 2013, 14, 601-607. [CrossRef] [PubMed]

83. Damiot, A.; Pinto, A.J.; Turner, J.E.; Gualano, B. Immunological Implications of Physical Inactivity among Older Adults during the COVID-19 Pandemic. Gerontology 2020, 66, 431-438. [CrossRef] [PubMed] 